\title{
Cortico-Striatal-Thalamic Loop Circuits of the Salience Network: A Central Pathway in Psychiatric Disease and Treatment
}

\author{
Sarah K. Peters ${ }^{1}$, Katharine Dunlop ${ }^{1}$ and Jonathan Downar ${ }^{1,2,3,4 *}$ \\ ${ }^{1}$ Institute of Medical Science, University of Toronto, Toronto, ON, Canada, ${ }^{2}$ Krembil Research Institute, University Health \\ Network, Toronto, ON, Canada, ${ }^{3}$ Department of Psychiatry, University of Toronto, Toronto, ON, Canada, ${ }^{4}$ MRI-Guided rTMS \\ Clinic, University Health Network, Toronto, ON, Canada
}

The salience network (SN) plays a central role in cognitive control by integrating sensory input to guide attention, attend to motivationally salient stimuli and recruit appropriate functional brain-behavior networks to modulate behavior. Mounting evidence suggests that disturbances in SN function underlie abnormalities in cognitive control and may be a common etiology underlying many psychiatric disorders. Such functional and anatomical abnormalities have been recently apparent in studies and meta-analyses of psychiatric illness using functional magnetic resonance imaging (fMRI) and voxelbased morphometry (VBM). Of particular importance, abnormal structure and function in major cortical nodes of the SN, the dorsal anterior cingulate cortex (dACC) and anterior insula (Al), have been observed as a common neurobiological substrate across a broad spectrum of psychiatric disorders. In addition to cortical nodes of the SN, the network's associated subcortical structures, including the dorsal striatum, mediodorsal thalamus

OPEN ACCESS

Edited by: Avishek Adhikari, Stanford University, USA

Reviewed by: Vikas Mishra, Louisiana State University Health Sciences Center, USA Stefano Delli Pizzi, University of Chieti-Pescara, Italy

*Correspondence: Jonathan Downar jonathan.downar@uhn.ca

Received: 14 November 2016 Accepted: 12 December 2016 Published: 27 December 2016

Citation:

Peters SK, Dunlop K and Downar J (2016) Cortico-Striatal-Thalamic Loop Circuits of the Salience Network: A Central Pathway in Psychiatric Disease and Treatment. Front. Syst. Neurosci. 10:104. doi: 10.3389/fnsys.2016.00104 and dopaminergic brainstem nuclei, comprise a discrete regulatory loop circuit. The SN's cortico-striato-thalamo-cortical loop increasingly appears to be central to mechanisms of cognitive control, as well as to a broad spectrum of psychiatric illnesses and their available treatments. Functional imbalances within the SN loop appear to impair cognitive control, and specifically may impair self-regulation of cognition, behavior and emotion, thereby leading to symptoms of psychiatric illness. Furthermore, treating such psychiatric illnesses using invasive or non-invasive brain stimulation techniques appears to modulate SN cortical-subcortical loop integrity, and these effects may be central to the therapeutic mechanisms of brain stimulation treatments in many psychiatric illnesses. Here, we review clinical and experimental evidence for abnormalities in SN cortico-striatal-thalamic loop circuits in major depression, substance use disorders (SUD), anxiety disorders, schizophrenia and eating disorders (ED). We also review emergent therapeutic evidence that novel invasive and non-invasive brain stimulation treatments may exert therapeutic effects by normalizing abnormalities in the $\mathrm{SN}$ loop, thereby restoring the capacity for cognitive control. Finally, we consider a series of promising directions for future investigations on the role of SN cortico-striatal-thalamic loop circuits in the pathophysiology and treatment of psychiatric disorders.

Keywords: corticostriatal, salience network, brain stimulation, depression, substance use disorders, anxiety disorders, repetitive transcranial magnetic stimulation 


\section{INTRODUCTION}

Psychiatric illnesses are among the leading causes of disability and disease burden worldwide in the 21 st century. For example, the 2010 Global Burden of Disease study identified major depressive disorder (MDD) as the second leading cause of years of life lost to disability (Ferrari et al., 2013). Overall, mental and substance use disorders (SUDs) accounted for $22.9 \%$ of the global burden of years of life lost to disability (Whiteford et al., 2013), with a prevalence of around one billion cases worldwide (Whiteford et al., 2015). These illnesses have high chronicity and community burden, and often show low response rates to existing treatments. For example, in major depression, conventional interventions are ineffective in at least one-third of patients, and relapse rates are high even when remission is achieved (Rush et al., 2006; Ferrari et al., 2013). Thus, an important task for basic and translational neuroscience is to better understand the underlying pathophysiology of psychiatric illness, and to develop treatments that effectively target this pathophysiology.

Over the last 25 years, one of the major advances in the field has been the development of increasingly detailed maps of the neural pathways that are affected in psychiatric illnesses. Steady progress is being made in localizing abnormalities of both brain structure and brain function. The neurologist's traditional question, "Where is the lesion?", formerly had few well-defined answers for most psychiatric disorders. However, today there is at least a first approximation of an answer to this question for many of the most prevalent types of mental illness. Progress in localizing psychiatric neuropathology has come from advances in non-invasive neuroimaging techniques suitable for in vivo use in humans. These include structural imaging techniques for mapping gray and white matter pathology, such as voxel-based morphometry (VBM) and diffusion tensor imaging (DTI), as well as functional imaging techniques including functional magnetic resonance imaging (fMRI), and positron emission tomography (PET).

In healthy control subjects, application of these brainimaging techniques has been especially fruitful for delineating the overall functional architecture of the human brain. One major discovery has been that brain activity, during tasks or at rest, is organized into functional networks of regions showing correlated activity over time. The networks themselves appear to be fairly consistent across individuals, with one influential report identifying a set of seven reliably reproducible major networks, subdivisible into a finer set of 17 smaller subnetworks (Yeo et al., 2011). The earliest description of resting-state networks was in the motor cortex (Biswal et al., 1995). Since that time, an extensive literature of thousands of publications has developed to describe the properties of several other major networks: a default-mode network most active during non-task cognitive states such as rumination or prospection (Raichle, 2015); a central executive network most active during performance of cognitive tasks involving attention or working memory (Bressler and Menon, 2010); and more circumscribed networks restricted to somatomotor or visual brain regions (Yeo et al., 2011).
Among these brain networks, one in particular is emerging as having particular significance to psychiatric illness: the salience network (SN; Seeley et al., 2007; Menon, 2011). Sometimes known by other terms such as the cingulo-opercular network (Dosenbach et al., 2008), the SN corresponds to the more anterior of the two subnetworks of the "ventral attention network (VAN)" described by Yeo et al. (2011). The SN features core nodes in the dorsal anterior cingulate cortex (dACC) and the bilateral anterior insula (AI), as well as additional cortical notes in specific regions of the dorsolateral prefrontal cortex (dlPFC) and inferior parietal lobule (IPL; Figure 1). In addition to these cortical nodes, the SN also includes a specific set of subcortical nodes in the head of the caudate nucleus, the mediodorsal nucleus of the thalamus (MDN) and dopaminergic brainstem nuclei (Menon, 2011). Together, these structures complete a discrete corticostriatal-thalamic-cortical (CSTC) loop that can be discerned using structural or functional neuroimaging (Seeley et al., 2007; Metzger et al., 2010), and that also corresponds to an analogous loop evident on tract-tracing studies in nonhuman primates (Choi et al., 2016).

In healthy brain function, cortical nodes of the $\mathrm{SN}$ may be activated passively by "salient" sensory stimuli (i.e., stimuli that draw attention for being unexpected, novel or behaviorally relevant; Corbetta et al., 2000; Downar et al., 2000, 2001, 2002). These cortical activations become accompanied by subcortical activations of the entire cortico-striatal-thalamic loop circuit during active, voluntary engagement of cognitive control, response selection or response inhibition; common examples include the Stroop task, Go/No-Go task or flanker interference task (Figure 2). As such, the SN has been proposed to play a key role in cognitive control (Ham et al., 2013), i.e., switching brain activity between introspective, ruminative functions of the default-mode network and externally focused, task-based functions of the central executive network (Menon and Uddin, 2010).

The importance of the $\mathrm{SN}$ to psychiatric illness emerges from an influential meta-analysis of $193 \mathrm{VBM}$ studies enrolling more than 7000 individuals with a wide variety of psychiatric diagnoses including depression, bipolar disorder, schizophrenia, SUD, obsessive-compulsive disorder (OCD) and anxiety disorders (Goodkind et al., 2015). Examining the patterns of decreased gray matter in each disorder, the authors found a common substrate across all diagnoses: loss of gray matter in the dACC and bilateral AI. The loci of gray matter loss corresponded closely with the core cortical nodes of the SN. As such, loss of structural and functional integrity in the $\mathrm{SN}$, and a resultant impairment of cognitive control, has been proposed as a transdiagnostic feature across many psychiatric illnesses (McTeague et al., 2016) and also as a target for novel therapeutic interventions, such as brain stimulation (Downar et al., 2016).

As of this writing, over 1500 publications have made reference to the $\mathrm{SN}$, and a number of excellent reviews are available to summarize this literature (Menon and Uddin, 2010; Menon, 2011; Dutta et al., 2014), including a recent book devoted entirely to the subject (Uddin, 2017). The present review article aims to build upon this work by considering the SN not just as 
A

Response Inhibition

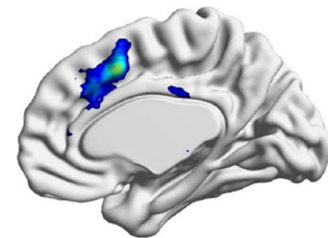

Response Selection

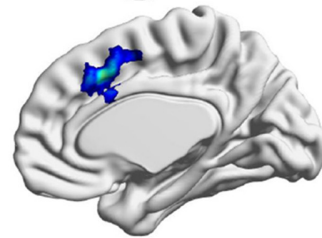

Salience Network

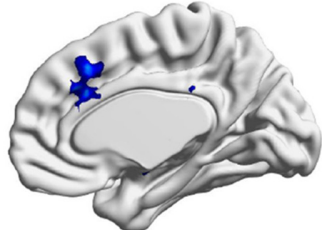

B

$\mathbf{R}$
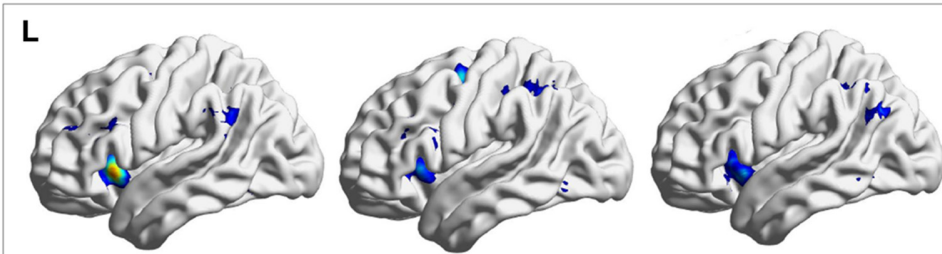

$\mathbf{R}$

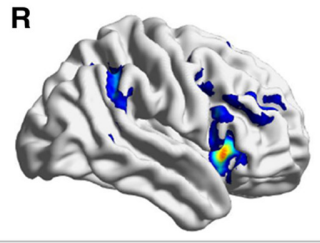

Response Inhibition

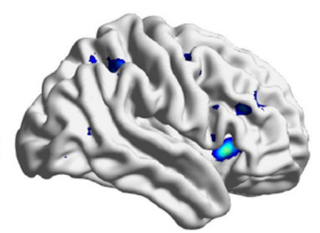

Response Selection

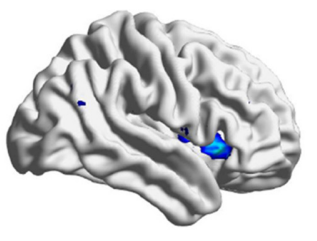

Salience Network

FIGURE 1 | Meta-analytic co-activation of salience network (SN) nodes for response selection and inhibition. Neurosynth meta-analytic results following key word searches for "response inhibition", "response selection" and "SN". Considerable overlap exists in the dorsal anterior cingulate cortex (dACC) region (A) and lateral parietal cortex (B).

a resting-state cortical network, but more specifically as an integrated, CSTC network with a particular role in the voluntary engagement of cognitive control. First, we examine the anatomy and function of the SN-CSTC in the healthy state. Second, we review the available literature on abnormalities of the SN-CSTC across a range of psychiatric disorders. Third, we review the available literature on how brain stimulation techniques can modulate the activity of the SN-CSTC, and how this modulation may achieve therapeutic effects in psychiatric illness. Finally, we consider a series of promising directions for future investigations

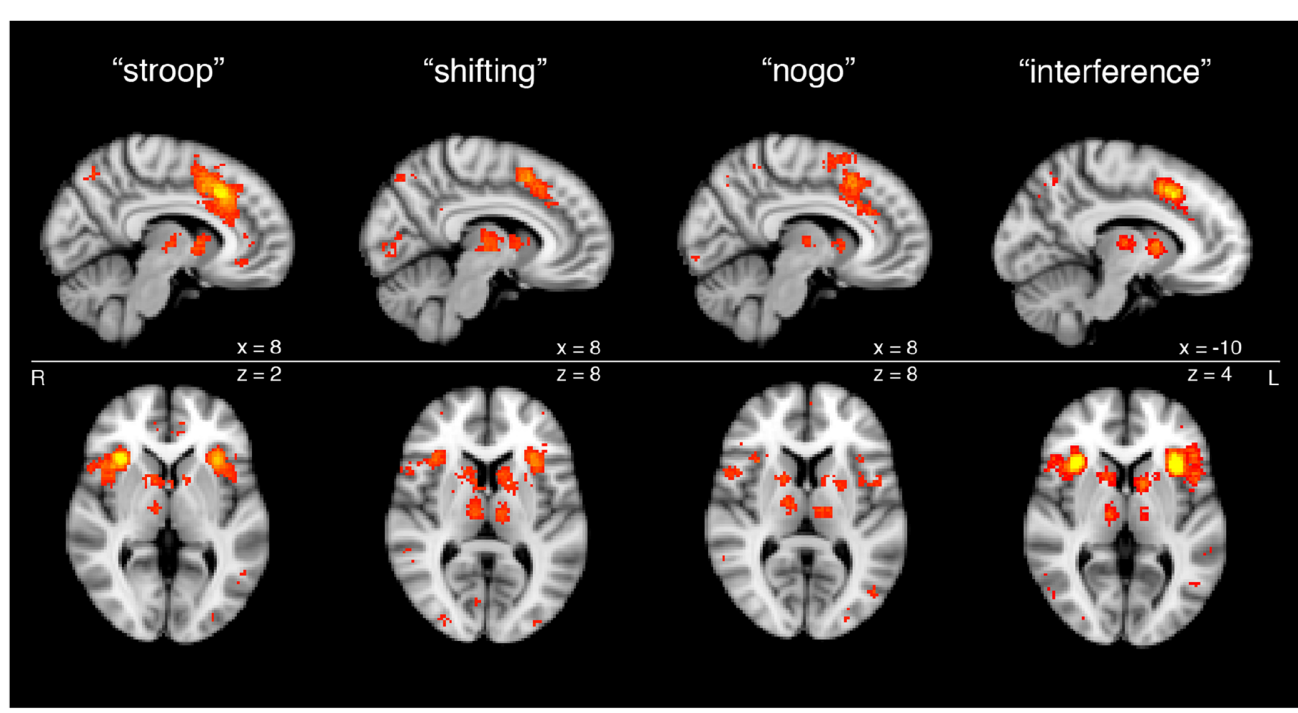

FIGURE 2 | Cortical and subcortical nodes of the SN from meta-analyses of functional magnetic resonance imaging (fMRI) studies of task-based activation during cognitive control. Subcortical components of SN processing are apparent across a variety of executive functioning tasks, including Stroop task, set shifting task, Go-No Go task and Flanker interference task, as shown through meta-analytic functional imaging data. 
on the role of the SN-CSTC loop circuits in the pathophysiology and treatment of psychiatric disorders.

\section{ANATOMY AND FUNCTION OF THE SN CORTICO-STRIATAL LOOP}

\section{Anatomy of the SN Cortico-Striatal-Thalamic Loop}

The cortical nodes of the SN are evident in one of the 17 restingstate networks cataloged in the 1000-subject, connectivity-based parcellation of resting-state fMRI data by Yeo et al. (2011). This network is the more anterior of the two subnetworks that comprise the larger VAN. While the more posterior subnetwork includes posterior insula and mid-cingulate nodes, the more anterior subnetwork includes adjacent AI and dACC regions (Figure 1). In addition, this network includes nodes in a specific region of the dlPFC, on the middle frontal gyrus, distinct from other lateral prefrontal regions assigned to the "frontoparietal" and "dorsal attention" networks in the Yeo et al. (2011) catalog. The SN also includes a specific region of the IPL, in the angular gyrus.

The interested reader can readily perform an independent replication of this map of regions by using the online, automated meta-analytic tool Neurosynth (Yarkoni et al., 2011; Figure 2). Entering the term "SN" yields a set of cortical regions that overlaps very closely with the more anterior cingulo-insular network of Yeo et al. (2011). Entering the term "cognitive control" - a core domain within the National Institute of Mental Health's proposed Research Domain Criteria for psychopathology research (Insel, 2014)—or associated terms for the subdomains of "response inhibition" or "response selection" likewise yields very a similar set of regions. Note that the purpose of this exercise is not to infer a particular functional role for these regions, as such inferences, based on correlations alone, are vulnerable to validity challenges. Instead, this meta-analytic exercise demonstrates the consistent co-activation of these regions as a coherent network across a variety of studies in the neuroimaging literature, with the functions of this network to be discussed subsequently.

The meta-analytic maps in Figure 2 are also notable because they reveal distinct subcortical nodes co-activating with the cortical nodes of the SN during the performance of active tasks, as opposed to during the resting state, from which the maps of Yeo et al. (2011) were derived. These subcortical nodes are present in the head of the caudate nucleus bilaterally, as well as in the thalamic MDN. These striatal and thalamic nodes, taken together with the cortical nodes of the SN, comprise the complete and functionally integrated CSTC loop circuit particular to the SN, for the purposes of the remainder of our review article.

\section{The SN CSTC Loop Circuit in its Classical Neuroanatomical Context}

The classical description of cortico-striatal-thalamo-cortical loop circuits of Alexander et al. (1986) included five parallel, functionally segregated circuits: (i) a motor CSTC loop through the supplementary motor area, putamen, ventrolateral globus pallidus interna (GPi) and ventrolateral thalamus; (ii) an oculomotor CSTC loop from the frontal eye field to the body of the caudate, caudomedial GPi and lateral ventral anterior thalamus; (iii) a lateral orbitofrontal CSTC loop from the lateral orbitofrontal cortex to the ventromedial caudate, medial dorsomedial GPi and medial ventral anterior thalamus; (iv) a dorsolateral prefrontal CSTC loop from the dlPFC to the dorsolateral caudate, lateral dorsomedial GPi and parvocellular mediodorsal thalamus; and (v) an anterior cingulate CSTC loop from the dorsal ACC to the ventral striatum, ventral pallidum and posteriomedial mediodorsal thalamus.

This classical set of five CSTC loops was originally derived from nonhuman primate work; in humans, a more complex and numerous set of CSTC loops is now considered likely. Indeed, a 1000-subject parcellation of resting-state functional connectivity in the striatum rather than the cortex (Choi et al., 2012) revealed substantially more than five distinct striatal regions, each associated with a specific cortical network cataloged in the parcellation of Yeo et al. (2011). As such, the CSTC loop of the SN may be expected to contain features of multiple classical loop circuits, rather than corresponding precisely to just one of them. The SN loop contains nodes that appear in the classical dorsolateral prefrontal CSTC loop (i.e., dlPFC, dorsolateral caudate and mediodorsal thalamus nodes), but also contains cortical nodes in the anterior cingulate and the AI, which do not appear in any of the classically described loops.

Conversely, each of the classically described CSTC loops of Alexander et al. (1986) also includes a specific subregion of the substantia nigra: rostrolateral for the dlPFC, and rostrodorsal for the anterior cingulate. Substantia nigra activations do not appear in the meta-analytic renderings of the SN (Figure 2), as may be expected given the limited spatial resolution of the fMRI data from which these renderings are derived. However, it should be noted that the substantia nigra and other midbrain dopaminergic centers such as the ventral tegmental area (VTA) are important contributors to all CSTC loops, and that rostral substantia nigra cell populations have been identified as key contributors to the CSTC loops serving SN cortical regions even in classical descriptions (Alexander et al., 1986).

\section{Functional Roles of Specific SN-CSTC Nodes}

Understanding the importance of SN function in cognitive control requires a thorough review of the broader functional roles of the SN's individual cortical and subcortical components. As noted earlier, key pre-Rolandic cortical nodes of the SN include the bilateral AI, dACC and dlPFC (Seeley et al., 2007). Separately, each of these areas have been linked to self-awareness, body perception (Craig, 2009) and fundamental cognition (Delevich et al., 2015). The AI plays an integral role in the response and experience of emotional states (Craig, 2003), and level of activity has been shown to correlate with stimulus valence (Anders et al., 2004). In addition to emotional perception, the 
AI is proposed to estimate changing environmental demands to modulate flexible cognitive control (Jiang et al., 2015). The AI also responds during action selection during decision-making (Paulus and Stein, 2006), and is therefore an integral component of cognitive control and the SN. Furthermore, the dACC has separately been implicated in cognitive regulation (Delevich et al., 2015), divergent thinking, error detection and response selection (Abraham et al., 2012; Sun et al., 2016). The dlPFC has extensive projections to striatal nuclei and is involved in top-down modulation of goal-directed behavior (Furman et al., 2011). The dlPFC is also a key hub of cognitive control, as it is implicated in executive function (Kuo and Nitsche, 2012) and has been identified as a dominant cortical area within the brain's central cognitive control network (Menon, 2011; McTeague et al., 2016).

Co-activation among cortical regions of the $\mathrm{SN}$ is also associated with cognitive and behavioral phenomena related to decision-making and cognitive control. For one, the AI, dACC and dIPFC activate synchronously in response to uncertainty in the environment; these regions overlap with areas implicated in negative mood states (Feinstein et al., 2006; Naqvi and Bechara, 2009; Davis and Hasson, 2016). The dACC and AI activate together during decision-making; co-activation has been shown to increase with task difficulty and stimulus ambiguity. This finding suggests that the "difficulty dependent functional architecture" between the AACC and AI plays a role in cognition by filtering and integrating internal and external stimuli during cognitive tasks (Lamichhane et al., 2016). In addition to coactivity with separate functional networks, the dACC also possesses extensive cortico-cortical connections within the PFC, including the cognitive dlPFC hub and premotor regions, placing it at the crossroads of learning and behavior systems (Haber, 2016).

Like other networks containing regions of association cortex, the $\mathrm{SN}$ links to subcortical nodes in areas of the striatum and limbic system, including (as noted above) the MDN, the dorsal striatum and dopaminergic nuclei within the midbrain and brainstem. The striatum, along with the entire basal ganglia, is important in coordinating and sequencing the diverse functions of the frontal lobes, from goal formation to executive function to cognition and the selection of specific actions and movements (Schultz et al., 2003). Whereas the ventral striatum is involved in reward and motivation, the central and dorsal striatum-including the caudate nucleus and the putamen-play more integral roles in cognition and executive function (Haber, 2016).

Within the thalamus, the $\mathrm{MDN}$ is a relay nucleus with reciprocal connections to regions of the medial PFC (mPFC) that assists in flexible action selection by integrating information from cortical, limbic and basal ganglia regions (Delevich et al., 2015). Loss of functional communication between the MDN and $\mathrm{mPFC}$ due to physical or chemical lesions has been shown to interrupt behavioral flexibility (Parnaudeau et al., 2013), learning and decision-making in both humans and animals (Mitchell, 2015). The MDN is integral to rapid associative learning and other executive tasks that involve complex cognition, though its precise role in integration and cognition remains incompletely characterized (Mitchell, 2015).

The role of the $\mathrm{SN}$ as a coordinator of other networks for cognitive control is supported by coactivation of its cortical and subcortical nodes during tasks. For example, functional links between the insula, which predicts changing cognitive control demand, and "classic" cognitive control circuits rooted in the $\mathrm{dACC}$ and dlPFC, allow for reactive attentional control (Jiang et al., 2015). Extensive connections from these regions to the dorsal striatum may link reactional cognitive control to behavioral guidance, allowing for on-line behavior regulation in response to salient environmental stimuli (Botvinick et al., 2004; Haber, 2016). Specifically, the dACC projects primarily to the dorsal caudate nucleus and ventral striatum, overlapping partially but not completely with frontostriatal projections from the dlPFC (Haber, 2016). Strengthened resting state functional connectivity between the AI and dACC has been associated with enhanced cue reactivity in other brain areas including the putamen, suggesting that functional connections throughout the loop allow incoming information to exert downstream effects on modulatory striatal areas (Janes et al., 2015). Although a general topographic organization exists between the frontal cortex and subcortical targets, there exists a complex convergence across CSTC loops that originate in prefrontal areas, including the ventromedial PFC (vmPFC) and orbitofrontal cortex; this overlap suggests that CSTC circuit integration is structural as well as functional, and provides modulation between and across reward, prediction and saliency circuits (Averbeck et al., 2014; Haber, 2016).

At the level of the brainstem, dopaminergic regions also play a significant modulatory role in the SN CSTC loop. Dopamine is integral to $\mathrm{SN}$ function: as noted earlier, the VTA and rostral substantia nigra project to the basal ganglia regions that subserve the classically described CSTC loops serving the anterior cingulate and dlFPC; these dopaminergic projections consequently play important roles in SN activity and modulation. Dopamine projections throughout the SN have been noted to play important roles not only in reward-oriented learning and goal-directed behavior, but also in processing motivational stimuli by directing attention to positive, adaptive or rewarding environmental stimuli (i.e., mediating the salience of environmental stimuli; Berridge, 1996; Koob and Volkow, 2010; Kroemer et al., 2014). Dopaminergic neurons in the midbrain are critical to CSTC loops that encompass integrated learning, executive function and motor control. Importantly, the mesolimbic and nigrostriatal dopaminergic pathway have been implicated in the encoding of "saliency prediction errors", underscoring their broader contributions to the attribution of salience to environmental stimuli (Kapur, 2003; Haber, 2016).

\section{An SN Architecture for Salience, Cognitive Control and Response Selection}

In summary, the SN CSTC loop appears to function as a distinct, well-integrated regulatory circuit that links cortical and subcortical regions involved in cognition, attentional control, 
motivation, motor control and salience. The SN CSTC loop has been described as a cortical input "filter" that selectively identifies and flags stimuli on which to base cognitive and behavioral responses (Furman et al., 2011; Choi et al., 2012). The dlPFC primarily projects to the dorsolateral caudate to serve cognitive control and executive functioning (Furman et al., 2011), while the thalamic MDN supports cortico-striatal-cortical information transfer and modulates cortical activity through extensive connections with the PFC and midbrain dopaminergic regions (Mitchell, 2015). Dopaminergic nuclei in the midbrain and brainstem, which are often associated with limbic circuitry, project widely throughout multiple loops of the striatum to serve a variety of functions, both structurally and functionally. For example, the substantia nigra makes extensive connections with the striatum, cortex, thalamus and neighboring brainstem regions, allowing dopaminergic outputs to exert far-reaching effects on the flow of CSTC information (Haber, 2016). At the same time, however, distinct populations of neurons in the rostral substantia nigra may project more specifically to the CSTC loops serving the cortical regions of the $\mathrm{SN}$, as recognized in classical descriptions of CSTC loop architecture (Alexander et al., 1986). Working together, the subcortical components of the SN communicate extensively with their cortical counterparts to select salient (motivationally relevant) stimuli, and to enable such stimuli to direct cognition and behavior. It is this architecture that enables the $\mathrm{SN}$ to play its central roles in salience detection, cognitive control and the selection and/or inhibition of behavioral responses during healthy brain function.

\section{ABNORMALITIES OF SN-CSTC LOOP CIRCUITS IN PSYCHIATRIC ILLNESSES}

Aberrations of corticostriatal loop circuits that modulate cognitive control and goal-directed behavior may underlie the pathophysiology of several psychiatric illnesses. Although specific psychiatric disorders, such as depression and schizophrenia are characterized by distinct constellations of symptoms, structural and functional abnormalities appear throughout similar nodes and networks transdiagnostically, across many Axis I disorders (Goodkind et al., 2015). It is possible that a spectrum of $\mathrm{SN}$ aberrations, ranging from hypo- to hyperactivity through the CSTC loop, can trigger a broad range of disabling behavioral and cognitive symptoms that are reflective of disrupted cognitive-attentional processing. The following section briefly explores the potential involvement of such corticostriatal circuits in the etiology, manifestation and treatment of major psychiatric disorders, including MDD, SUD, anxiety disorders including OCD and post-traumatic stress disorder (PTSD), schizophrenia and eating disorders (ED).

\section{Major Depressive Disorder (MDD)}

MDD is characterized by persistent low mood, accompanied by loss of interest and pleasure, increased fatigue and irritability and difficulty concentrating and making decisions (Benazzi, 2006). Neuroimaging analyses of individuals with MDD have revealed both anatomical and functional differences throughout SN nodes relative to healthy controls. Anatomically, investigations have revealed reduced gray matter volume in the anterior cingulate cortex, caudate nucleus, putamen (Bora et al., 2012; Shepherd, 2013). Thalamic volume abnormalities are mixed (Webb et al., 2014; Zhao et al., 2014; Hagan et al., 2015). Neurochemical ligands via PET have shown reduced midbrain and subcortical serotonergic receptor binding in MDD (Hahn et al., 2014; Yeh et al., 2015).

While anatomical and neurochemical differences have been identified in SN nodes, symptoms of MDD have often been attributed to dysfunctional ventral CSTC loops rather than volumetric deviations alone (Bora et al., 2012; Kerestes et al., 2015). MDD patients generally exhibit reduced sensitivity to reward; this cognitive blunting is reflected by reduced ventral CSTC loop activation in the orbitofrontal cortex, anterior cingulate and ventral striatum to reward and loss receipt/anticipation (Smoski et al., 2011; Chantiluke et al., 2012; Schiller et al., 2013; Admon et al., 2015; Manelis et al., 2016).

Although MDD has classically been described in terms of dysfunctional ventral corticostriatal loops that play a role in affect and reward, recent reports have identified abnormalities within dorsal, cognitive corticostriatal loops in MDD patients (Kerestes et al., 2015). Specifically, individuals with depression are reported to show increased functional connectivity between the dorsal caudate nucleus and right dIPFC, with disease severity correlated to increased connectivity; hyperactivation between these regions may indicate a pathological compensation of cognitive processing over negative emotions and stimuli (Furman et al., 2011; Kerestes et al., 2015).

In addition to $\mathrm{SN}$ structural and functional aberrations in MDD, symptomatic improvement to conventional treatment is predicted by differential SN function. Connectivity between the putamen, caudate and cingulate during a monetary incentive delay task has been shown to predict response to psychotherapy (Admon et al., 2015; Walsh et al., 2016). High baseline ACC and low striatal activity were also shown to be predictive of improvement on antidepressants in a recent meta-analysis of 15 studies (Fu et al., 2013).

SN function change is also attributed to symptomatic improvement to conventional interventions. A recent meta-analysis based on nine MDD fMRI studies established that antidepressant treatment resulted in increased activation in the dIPFC, AI and ACC, and decreased activation in the thalamus and caudate nucleus, during emotional processing, representing normalization of resting state activity (Delaveau et al., 2011). Similar analyses consistently identify functional increases in these areas relative to baseline following SSRI treatment based on functional activity during negative mood induction (Fitzgerald et al., 2008) and incentive cue processing (Stoy et al., 2011).

MDD patients vary widely in terms of symptom presentation; recent work has sought to characterize MDD by distinct symptomatic and neural subtypes, or "endophenotypes". In one notable study, MDD was partitioned into three subtypes, one of which was characterized by regulatory deficits in 
cognitive control and altered electrical current densities in nodes of the SN, specifically the dIPFC and dACC (Webb et al., 2016). The conflation of several hypothetically heterogenous MDD pathophysiological profiles in functional studies to date may obscure important characteristics that differentiate one or more of these subtypes. Future work should further characterize the symptomatic and neural heterogeneity of MDD into reliable endophenotypes.

\section{Substance-Use Disorders (SUDs)}

SUDs involve abnormal reward and motivational responses to salient drug-related cues in the environment. Impulsivity and compulsivity are well-characterized traits of SUD, and both traits play important roles in the addiction cycle (Koob and Volkow, 2010). Traditional neurobiological research in SUD has attempted to identify how subcortical and dopaminergic systems affect drug seeking behavior (Menon, 2011). For example, combined PET-fMRI data suggests that frontostriatal abnormalities may be associated with a reduction of striatal D2 dopamine receptors and prefrontal, dlPFC metabolic activity (Tomasi and Volkow, 2013).

Structurally, nodes of the SN display volumetric abnormalities in SUD. For example, cocaine users display altered cingulate, insula and caudate volume (Ersche et al., 2011; Moreno-López et al., 2012). Also, methamphetamine craving was associated with volumetric differences in the insula, PFC and thalamus; this association was further correlated with dopaminergic receptor availability in the midbrain (Morales et al., 2015). Functional abnormalities of single SN nodes have been related to SUD behaviors; the AI is considered critical to conscious drug-seeking motivation (Naqvi and Bechara, 2009).

$\mathrm{SN}$ network connectivity is implicated in SUD. For example, lower resting-state functional connectivity between the striatum, cingulate and insula have been found in cocaine users; dorsal ACC and dlPFC connectivity was linked to loss of control and impulsivity, respectively ( $\mathrm{Hu}$ et al., 2015). Furthermore, reduced activation in the dorsal caudate and dlPFC has been found to be correlated with impulsivity and Stroop task errors in SUD (Qiu et al., 2013; Feng et al., 2016; Yuan et al., 2016). Conversely, gambling addiction has displayed increased PFC connectivity to the striatum; this connectivity was associated with impulsivity, smoking frequency and craving severity (Koehler et al., 2013). Stronger functional coherence within SN cortical nodes was recently linked to enhanced reactivity to smoking cues in individuals with nicotine dependance, suggesting that hyperconnectivity within the SN may be responsible for increased cue reactivity (Janes et al., 2015).

On task-based fMRI, another study used a reward-guided decision-making task to investigate reward prediction errors-signals that guide learning behaviors-in men with alcohol addiction. SUD patients also demonstrated abnormal dlPFC-striatal functional connectivity following "wins", and "losses", suggesting that learning mechanisms are impaired due to disrupted regulation of frontostriatal interactions (Park et al.,
2010). Similarly, reduced response to reward is observed across the striatum, mPFC and dlPFC (Forbes et al., 2014).

Additionally, it appears that addictive substances activate dopamine-dependent saliency systems in the brain, resulting in the replacement of adaptive stimuli by more salient drug-related cues (Koob and Volkow, 2010). For example, higher dorsal caudate reactivity during cue induced craving has been found in heavy drinkers, while ventral striatal activity was found in lighter drinkers, and this the functional difference between drinkers was correlated with compulsive craving severity (VollstädtKlein et al., 2010). In another study, higher caudate and ACC activity during cue-induced craving predicted a transition to heavier drinking habits (Dager et al., 2014). Increases in compulsive drug-seeking behavior has been hypothesized to represent a shift from "top-down", prefrontal cortical behavior control to striatal behavioral control due to progressively altered dopamine transmission (Goldstein et al., 2009; Koob and Volkow, 2010).

In summary, drug addiction has been described as a process of maladaptive neuroplasticity that begins in mesolimbic reward circuits and emanates through interconnected loops in the dorsal striatum, eventually cascading to the cortex where dysregulation results in permanent changes to habitual behavior and impulsivity (Koob and Volkow, 2010). SN frontostriatal circuit integrity may be associated with improved impulse control (Peper et al., 2013); conversely, improved top-down cognitive control over the striatum by prefrontal cortical regions may reduce impulsivity (Peters and Büchel, 2011).

\section{Obsessive-Compulsive Disorder (OCD)}

OCD is classically characterized by intrusive thoughts (obsessions) that are distressing and disruptive to normal function, followed by repetitive actions (compulsions) aimed at reducing the associated distress (American Psychiatric Association, 1994). In more than half of individuals with OCD, these symptoms may be severe enough to cause absence from work, school or social engagements; further, treatment of these severe cases is often more difficult (Bourne et al., 2012). Importantly, obsessions and compulsions represent dysfunctional cognitive and motor processes that become repetitive and disturbing; it is suggested that hyperactivation within CSTC circuits and changes to CSTC topography may underlie such ritualistic thoughts and behaviors (Graybiel and Rauch, 2000; Nakamae et al., 2014).

OCD has traditionally been attributed to dysfunction within orbitofronto-CSTC loops; despite being somewhat distinct from CSTC loops that traditionally mediate the cognitive domain, this dysfunction is hypothesized to contribute to the unusual cognitive control of behavioral patterns often observed in OCD (Graybiel and Rauch, 2000; Dunlop et al., 2016). CSTC loop circuits are also relevant to OCD due to their integral role in emotional, cognitive and behavioral self-regulation (Marsh et al., 2009a). Results of a 7-study meta-analysis examining OCD resting-state fMRI suggested 
OCD tends to most reliably demonstrate corticostriatal network abnormalities relative to other anxiety disorders (Peterson et al., 2014). While many symptoms of OCD are related to disruptions within orbitofronto-CSTC loops, regions of the SN likely play a large role in specific symptoms; OCD symptoms.

Within nodes of the $\mathrm{SN}$, volumetric changes have been identified in the ACC, striatum and thalamus (Gilbert et al., 2008; Hoexter et al., 2012; Hou et al., 2013; Atmaca et al., 2016). Functionally, the ACC appears to be abnormally altered during disease-relevant tasks. For example, OCD patients experience ACC hyperactivity during symptom aggravation and while committing errors during a flanker interference task (Bourne et al., 2012). Even outside of active task engagement, nodes of the SN are abnormally hyperactive: increased resting state functional activity of the ACC, MDN (Rauch et al., 2006a; Bourne et al., 2012), dlPFC (Figee et al., 2014) and caudate nucleus (Graybiel and Rauch, 2000) has been noted in individuals with OCD. Frontostriatal functional connectivity stemming from the dlPFC (Vaghi et al., 2016), and altered striatal connectivity to the insula (Bernstein et al., 2016) has been linked to OCD severity.

Interestingly, functional changes within $\mathrm{SN}$ nodes are related to OCD symptom improvement. Notably, stereotaxically targeted lesions of the dACC during cingulotomy procedures results in symptom improvement in some individuals with treatment-resistant OCD (Dougherty et al., 2002); such lesions also reduce glucose metabolism in the caudate, medial dorsal thalamus and caudate (Zuo et al., 2013). Traditional pharmacotherapies are shown to attenuate ACC and striatal hyperactivity (Rauch et al., 2006a), and normalize thalamic, cingulate and putamen gray matter volume (Hoexter et al., 2012; Atmaca et al., 2016). On resting-state fMRI, reduced dorsal caudate-anterior thalamus functional connectivity was associated with symptomatic improvement following fluoxetine (Anticevic et al., 2014). Such findings highlight the importance of SN-CSTC dysfunction in the pathophysiology of OCD.

\section{Post-Traumatic Stress Disorder (PTSD)}

PTSD occurs in approximately $10 \%$ of individuals who have experienced a traumatic event; symptoms include intrusive re-experiencing of the traumatic event, and avoidance behaviors in order to escape similar or triggering situations (Koch et al., 2016). Changes in arousal, behavior and mood, as well as impairments in executive functioning, are also commonly seen in individuals with PTSD (American Psychiatric Association, 2013). Structurally, PTSD patients exhibit reduced gray matter volume in $\mathrm{SN}$ regions including the $\mathrm{ACC}$, striatum and insula (Meng et al., 2016); caudate and insular volumetric reductions correlate with PTSD severity (Herringa et al., 2012). Cortical thinning has also been observed in the rostral ACC and insula, and disrupted structurally connectivity is seen between the ACC, thalamus and insula in PTSD (Mueller et al., 2015).

There is evidence that symptoms associated with PTSD involve potential changes to both singular SN nodes and corticostriatal circuits. First, during symptom provocation, PTSD symptom severity correlates with ACC activity, and heightened activity variability in the insula and ACC, and lower variability in activity in the dlPFC and striatum (Ke et al., 2015). Another study also found increased connectivity between the insula, mPFC, striatum and dorsal ACC during symptom provocation (Cisler et al., 2014). Second, generalization of fear associations in PTSD is associated with heightened insula and thalamus activity (Morey et al., 2015). Third, PTSD severity during an emotional Stroop task was associated with higher dlPFC, dmPFC and dorsal ACC activation to emotional stimulation (White et al., 2014). Finally, higher dorsal caudate and frontal activation during inhibitory control predicts response to CBT in $\mathrm{PTSD}$, suggesting a role of $\mathrm{SN}$ function in symptom improvement following psychotherapy (Falconer et al., 2013).

Resting state functional connectivity also has revealed abnormal SN functional connectivity in PTSD. One recent meta-analysis showed that PTSD patients display enhanced connectivity within the SN, suggesting excessive salience processing within the $\mathrm{SN}$ serves as a mechanism of abnormal hyper-monitoring the external environment (Koch et al., 2016). Similarly, individuals with PTSD demonstrate hyperactivity relative to controls within the AI and dACC across a variety of executive functioning and emotional processing tasks, in conjunction with reduced functional connectivity between regions involved in fear, stress and negative affect. It is possible that enhanced vigilance combined with decreased top-down cognitive control over fear responses underlies PTSD symptoms (Rauch et al., 2006b; Patel et al., 2012).

With regards to abnormalities throughout CSTC loops, individuals with PTSD have been shown to have reduced thalamic functional connectivity with a variety of brain regions including the rostral ACC and mPFC (Duarte et al., 2011; Yin et al., 2011), and it has been suggested that flashback symptoms occur in part due to dysfunctional corticothalamic activity (Liberzon et al., 1996/1997). Recently, the medial rostral dorsal caudate was identified as a site of convergence between the IPL, dIPFC and dACC, suggesting that striatal inputs that mediate and bias attentional salience and cognitive control (Choi et al., 2016). A region allowing for interaction of such cortical areas may be strongly implicated in the allocation of stimuli salience and environmental attention, and authors suggest that future investigations probe this area to learn more about its involvement and potential therapeutic value in disorders including PTSD (Choi et al., 2016).

\section{Schizophrenia}

Schizophrenia is a disabling psychiatric condition that manifests in primarily three domains: positive, negative and cognitive symptoms. Positive symptoms include additions to an individual's repertoire of thoughts or behavior, such as hallucinations or delusions, whereas negative symptoms refer to flattened affect, anhedonia or catatonic symptoms (American Psychiatric Association, 2013). Individuals with this disorder may experience impairments in executive function and have been found to have deficits in cognitive, but not motor, learning 
(Foerde et al., 2008; Parnaudeau et al., 2013). In addition to cognitive deficits, schizophrenia is defined by psychosis and psychotic episodes (American Psychiatric Association, 2013).

Structural abnormalities within the SN have been observed in schizophrenia, specifically between the insula, ACC, dlPFC and striatum (White et al., 2010; Menon, 2011; Quan et al., 2013; Iwabuchi et al., 2015; Chen et al., 2016). Cortical thinning is also observed in the AI, IFG and ACC; this volumetric change is reflected by altered functional connectivity ( $\mathrm{Pu}$ et al., 2012; Pujol et al., 2013). Further, schizophrenia has been associated with abnormal dopamine signaling within the cortex (Knable and Weinberger, 1997).

Psychosis and salience attribution deficits in schizophrenia are related to inter-network dysfunction between the $\mathrm{SN}$, CEN and DMN (Palaniyappan et al., 2011, 2013; Moran et al., 2013; Lee et al., 2014; for a comprehensive review, see Nekovarova et al., 2014). Patients experiencing auditory hallucinations show increases in $\mathrm{AI}$ and frontal operculum activation and altered SN dynamics, indicating that internally generated stimuli are perceived as abnormally salient (Sommer et al., 2008; Lefebvre et al., 2016). Indeed, AI resting-state functional connectivity is correlated to symptom severity (Manoliu et al., 2013).

Frontostriatal dysconnectivity between the dlPFC, dACC, AI and putamen are observed during an emotion judgment task, indicating that $\mathrm{SN}$ inappropriate assigns valence to emotionally salient stimuli (Lee et al., 2014). Additionally, certain genes that impart increased risk of developing schizophrenia have been associated with decreased striatal volume and hyperconnectivity within frontostriatal loops (Shepherd, 2013).

Of note, insular-SN connectivity can discriminate patients from healthy controls (Mikolas et al., 2016; Wang X. et al., 2016). One recent publication associated the severity and development of psychosis with the extent of hypoconnectivity throughout SN CSTC circuitry, specifically between the left $\mathrm{AI}$, the bilateral putamen, and caudate nucleus (Wang C. et al., 2016). Further, Wang C. et al. (2016) demonstrated that individuals with schizophrenia displayed reduced structural and functional integrity within CSTC tracts of the SN. Reductions in network integrity may also explain interrupted information processing observed in individuals with schizophrenia, which is experienced as abnormal sensory processing (White et al., 2010).

\section{Eating Disorders (EDs)}

EDs affect about $0.5 \%$ of women and can cause significant physical and psychological burden; for example, anorexia nervosa (AN) has the highest mortality of any psychiatric disorder (Hudson et al., 2007). EDs are characterized by altered self-image and maladaptive eating behaviors, and include $\mathrm{AN}$, bulimia nervosa $(\mathrm{BN})$ and binge-eating disorder (BED; McClelland et al., 2013). In recent years, functional imaging studies of EDs have pinpointed the intersection of cognitive and reward systems as integral to eating behavior regulation (ValLaillet et al., 2015).

One systematic review of 10 structural MRI studies suggested that compared to healthy controls, both decreased and increased gray matter volumes in a variety of frontal brain areas characterize $\mathrm{AN}$ and $\mathrm{BN}$, respectively, and may normalize with successful treatment (Van den Eynde et al., 2012). AN and BN patients show reduced gray matter volume specifically in $\mathrm{SN}$ regions including the caudate nucleus, anterior cingulate cortex and insula, among other areas (Schäfer et al., 2010; Friederich et al., 2012; Frank et al., 2013; Coutinho et al., 2015). On DTI, AN patients show abnormal thalamic connectivity to the dlPFC and anterior PFC (Frieling et al., 2012; Hayes et al., 2015), and reduced fractional anisotrophy in the medial dorsal thalamic radiations (Biezonski et al., 2015; Hayes et al., 2015). Further, increased structural connectivity between the insula and striatum is observed in ED (Frank et al., 2016; Shott et al., 2016).

On task-based fMRI, BN patients display low frontostriatal activity on a number of cognitive control tasks, including the Simon Spatial task (Marsh et al., 2009b, 2011) and Go/No-Go (Skunde et al., 2016). Similarly, AN patients have also been shown to have altered frontostriatal activation on executive function tasks, including the Wisconsin Card Sorting Task (LaoKaim et al., 2015), and delay discounting (Wierenga et al., 2014; Decker et al., 2015). During reward conditioning, AN patients show abnormally high SN activity relative to controls (Frank et al., 2012), and an increased preference for delayed rewards over immediate rewards. Dorsal caudate dysfunction is also associated with AN patients in a number of tasks, including a monetary choice task (Bischoff-Grethe et al., 2013; Bailer et al., 2016), and food-cue processing (Sanders et al., 2015). This increase in striatal reactivity may be related to a number of trait-based or neurochemical factors, including abnormal trait anxiety (Bailer et al., 2016), harm avoidance (Bailer et al., 2013), obsessive thoughts (Rothemund et al., 2011) and striatal dopaminergic receptor availability (Bailer et al., 2013, 2016; Broft et al., 2015).

Both $\mathrm{AN}$ and $\mathrm{BN}$ patients have altered $\mathrm{SN}$ function during disease-relevant stimuli. In one study, presenting food-related visual cues to individuals with either $\mathrm{BN}$ or $\mathrm{BED}$ resulted in increased ACC and insula activation, and BN patients reported higher levels of arousal (Schienle et al., 2009). In another study, BN participants displayed low activity in the insula to food images, while AN showed higher activity in the caudate and insula (Brooks et al., 2011). Another study showed that AN participants show abnormally high activation to aversive taste in the insula and putamen (Cowdrey et al., 2011). Abnormal ACC-insular resting state activity has also been observed in ED patients (Amianto et al., 2013; Dunlop et al., 2015b). Altered connectivity strength and path length between the insula and thalamus has been observed in $\mathrm{AN}$ (Geisler et al., 2016), as well as decreased functional connectivity between the thalamus, putamen and insula (Ehrlich et al., 2015). Given all this evidence, the variation in symptoms across different classes of EDs-for example, the presence of binging behavior in $\mathrm{BN}$ and $\mathrm{BED}$, but not $\mathrm{AN}$-make it difficult to assess convergent implicated brain regions, and both structural gray matter and functional activity analyses in these populations should be expanded (Schäfer et al., 2010). 
There is evidence that corticostriatal loop circuits are affected in EDs. Specifically, individuals with EDs often display impaired self-regulatory control-this includes inhibition of both motor and emotional responses-that can be traced to dysfunction within dorsal frontostriatal circuits crucial for self-regulation (Berner and Marsh, 2014). Authors who recently reviewed the balance between incentive reward and inhibitory circuits in EDs (Wierenga et al., 2014) hypothesized that different patterns of disordered eating represent a spectrum of regulatory capability, ranging from extreme cognitive control in $\mathrm{AN}$ to deficiencies of cognitive control in BN. The dorsal neural system supporting such regulatory capacity-which includes functions of inhibition, emotion regulation, and goal-directed behavior-includes the dorsal caudate, dACC and insula, among other SN regions (Wierenga et al., 2014).

\section{Summary}

Both structural and functional abnormalities within and between corticostriatal loop circuits are associated with psychiatric pathology. Generally, these disorders can be characterized by an inability to exert cognitive control over maladaptive thoughts, impulsive behaviors or attention to appropriate salient internal and external stimuli; for example, OCD involves disrupted control mechanisms, but lacks the element of disturbed motivational salience that is well defined in MDD and SUD. Regardless of clinical phenotype, consistent implication of the dACC, AI and dorsal striatal nodes in psychiatric etiology suggests that the SN-CSTC loop is indeed crucial for psychological health and adaptive functioning across a variety of disorders.

\section{TARGETING THE SN-CSTC WITH THERAPEUTIC BRAIN STIMULATION}

For individuals with psychiatric disorders, the mainstays of conventional treatment are psychotherapy and pharmacotherapy. However, these approaches are ineffective for a substantial proportion of patients. For example, an estimated one third of MDD patients do not respond to 2-4 sequential trials of pharmacotherapy or psychotherapy (Rush et al., 2006). Therapeutic brain stimulation is an emerging alternative in cases where conventional approaches fail (Figure 3). A number of techniques for therapeutic brain stimulation are entering clinical use for treatment-resistant psychiatric illnesses. These include deep brain stimulation (DBS; Lozano and Lipsman, 2013), repetitive transcranial magnetic stimulation (rTMS; Lefaucheur et al., 2014) and transcranial direct current stimulation (tDCS; Tortella et al., 2015).

For the neuroscientist, these techniques can serve a dual role as both an intervention and a probe for investigating human brain function, either in health or in psychiatric illness. It is possible to assess the neurophysiological effects of brain stimulation using neuroimaging techniques, complemented by electrophysiological recordings. As described above, many clinical features of psychiatric illnesses are rooted in altered activity within relevant brain networks. Given that brain stimulation treatments are usually targeted to a specific brain region, the goal of such a treatment is to normalize regional cortical and downstream network activity.

The SN has been proposed as a key target for neuromodulation treatments across a variety of psychiatric illnesses (Downar et al., 2016; Dunlop et al., 2016). Stimulationdriven changes in the SN-CSTC loop could restore capacity for cognitive control via changes in network activity, resulting in symptom reduction (Veit et al., 2012; Sale et al., 2015). With respect to $\mathrm{SN}$ nodes and their downstream regulatory loops, both invasive and non-invasive brain stimulation techniques appear capable of modulating the activity of these circuits by imposing long-term changes in cortical nodes that cascade through the SN loop. These changes are accompanied by alterations in affect and behavior in the patient receiving treatment (Di Filippo et al., 2009).

In this section, we review the effects of DBS, rTMS and tDCS on cortico-striatal-thalamo-cortical circuitry through the SN specifically, such as they are known at the present time. We also review the available evidence to date about how changes in SN-CSTC function relate to the therapeutic effects of neuromodulation therapies in psychiatric illness.

\section{Deep Brain Stimulation (DBS)}

DBS involves neurosurgical implantation of stimulation electrodes in target regions of the brain under stereotaxic guidance. The implanted electrodes modulate abnormal neural activity by applying electric fields that stimulate adjacent axons, resulting in the modification of electrical communication within connected functional brain networks (Kühn and Volkmann, 2016; Lin et al., 2016; Rachid, 2016). Although this technique is invasive, expensive and requires subspecialist expertise, DBS does carry the advantage of being able to directly target the deeper nodes of CSTC circuits, such as the striatum, pallidum, subthalamic nucleus or brainstem dopaminergic structures, as well as the white matter tracts connecting these nodes.

Some of the earliest uses of DBS were in modulating the activity of motor CSTC loops to treat movement disorders, including essential tremor and Parkinson's disease (PD; Chen et al., 2013); the latter remains the most common therapeutic application of DBS (Fasano and Lozano, 2015). However, more recently, DBS has begun to show promise as a treatment for a number of neuropsychiatric disorders (Williams and Okun, 2013), including MDD (Mayberg et al., 2005; Schlaepfer et al., 2014), OCD (Lipsman et al., 2013a) and AN (Lipsman et al., 2013b).

For DBS in psychiatric disorders, the stimulation targets are generally found in CSTC circuits outside of motor loop. In $\mathrm{MDD}$, for example, targeting the subgenual cingulate cortex (sgCC) may have modulatory downstream effects on the dACC by propagating stimulation effects through the rostral cingulate cortex (Morishita et al., 2014). Further, sgCC-DBS has normalized hypoactivity of the dlPFC and ACC, among other areas, in depressed patients without any observed cognitive side 


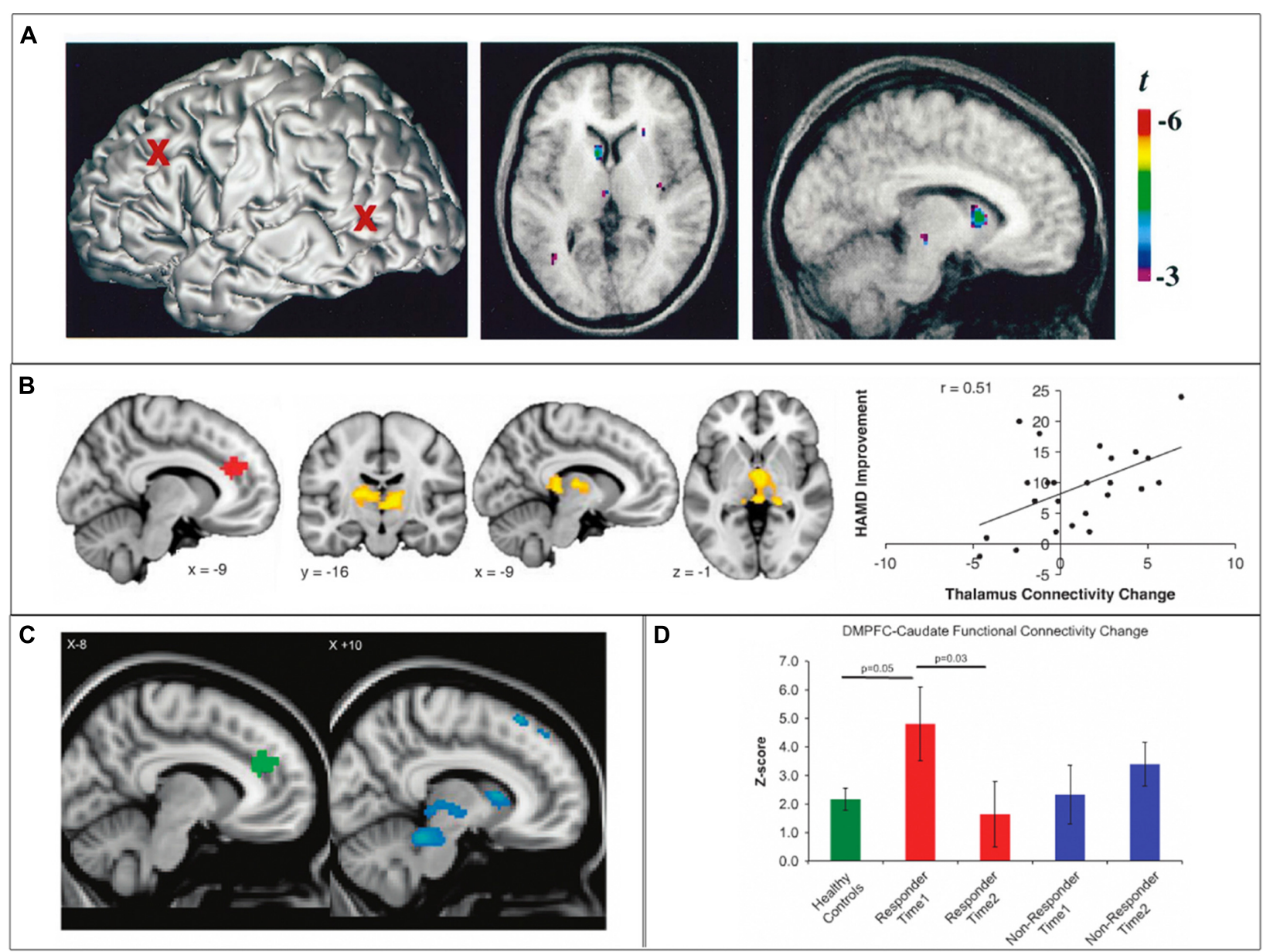

FIGURE 3 | SN cortico-striatal-thalamo-cortical circuit engagement during therapeutic brain stimulation. (A) In healthy controls, $10 \mathrm{~Hz}$ repetitive transcranial magnetic stimulation (rTMS) of the left dorsolateral prefrontal cortex (dIPFC) results in increased dopamine transmission in the ipsilateral caudate nucleus and thalamus. (B) In depressed individuals, clinical improvement following $10 \mathrm{~Hz}$ dmPFC-rTMS was correlated with increased connectivity between the dACC and the thalamus. (C,D) In obsessive-compulsive disorder (OCD) patients, clinical improvement following $10 \mathrm{~Hz}$ dmPFC-rTMS was correlated with decreased functional connectivity between the dACC and the caudate nucleus, thalamus, putamen and midbrain. Adapted from (A) Strafella et al. (2001); (B) Salomons et al. (2014); (C,D) Dunlop et al. (2016).

effects (Mayberg et al., 2005). More generally, sgCC-DBS appears to be an effective intervention for severe, treatment-resistant MDD. Follow-up studies and meta-analyses suggest that overall, sgCC-DBS may continue to ameliorate depressive symptoms over time, with persistence of beneficial effect for over a decade in some cases (Giacobbe et al., 2009; Morishita et al., 2014). It also appears that response rates to ongoing DBS stimulation may improve over time, with benefits accruing and persisting over periods of several years among responders (Kennedy et al., 2011).

Thus far, DBS has not been used to target the SN-CSTC loop directly in MDD. However, DBS targeting the nucleus accumbens (NAcc) has been successful in some cases of treatment-resistant depression, with responders showing improvements in hedonic capacity (Bewernick et al., 2010). PET imaging in these cases demonstrated that stimulation of the NAcc yielded reductions in metabolic activity in the "reward CSTC loop" projecting to vmPFC and frontal pole.
This finding illustrates the potential of DBS to modulate (i.e., suppress) activity in CSTC circuits for therapeutic effect.

One illustrative case study suggests that DBS targeting the SN-CSTC may not necessarily exert desirable effects in MDD (Stefurak et al., 2003). In this report, a patient with intractable PD (and a remote history of a major depressive episode) underwent implantation of DBS electrodes bilaterally in the subthalamic nucleus. Activation of one electrode yielded the expected improvement in the tremor of the contralateral upper limb. However, activation of the other electrode had the unexpected effect of inducing an intense dysphoria of rapid onset. The patient described feeling "similar in some respects to my depression but a thousand times worse... Someone could have come in to shoot me and I could not have cared less". The effect was reliably reproducible, and mood returned rapidly to baseline with cessation of stimulation. When functional neuroimaging was performed, 
stimulation of one electrode yielded reductions in supplementary motor area activity, alongside improvement of the contralateral tremor. Stimulation of the other electrode yielded suppression of activity in the $\mathrm{SN}$ regions including the dACC as well as the caudate nucleus, accompanied by rapid descent into dysphoria. This case illustrates the feasibility of using DBS to modulate the SN-CSTC loop circuit, and also illustrates that such modulation can be accomplished via deep subcortical targets such as the subthalamic nucleus. However, at the same time, it illustrates that suppression of SN activity may impair rather than improve mood regulation, with deleterious rather than beneficial effects in the setting of MDD.

The suppressive effects of subcortical DBS on CSTC loop functions may be of more benefit in the setting of OCD. DBS targets in OCD may include the ventral striatum, sgCC, NAcc or the medial forebrain bundle (Lipsman et al., 2013a; Williams and Okun, 2013). Although these targets lie outside the main nodes of the SN-CSTC loop, there is some evidence that the therapeutic effects of DBS in OCD ensue from modulation of activity in SN sites. Specifically, a case series of NAcc-DBS in OCD reported considerable inter-individual variability in the degree of clinical improvement; resting-state fMRI revealed that the degree of symptom improvement correlated to the degree of reduction in functional connectivity between the stimulation target (NAcc) and two nodes of the SN: the dACC and the dlPFC (Figee et al., 2014). Indeed, it has been suggested that all of the major DBS targets in OCD exert therapeutic effects by modulating activity in the ACC and associated regions of the striatum (Bourne et al., 2012).

Subcortical DBS for psychiatric disorders is thought to influence the regulatory activity of CSTC loops. Investigations of DBS to central thalamic regions in neurological disorders have identified several effects of stimulation, including increased D2 dopamine receptor concentration in the striatum, improved functional connectivity between the thalamus and striatum, and modulated plasticity in the striatum (Bourne et al., 2012). These effects are hypothesized to contribute to improvements in striatal plasticity, resulting in increased regulatory capacity over cognition and learning (Lin et al., 2016).

\section{Repetitive Transcranial Magnetic Stimulation (rTMS)}

rTMS is a non-invasive brain stimulation technique that alters neural excitability by delivering focused magnetic field pulses to cortical areas non-invasively through the skull (Hallett, 2007). Repeated trains of pulses can produce durable increases or decreases (depending on the stimulation pattern) in the strength of the synapses of the stimulated neurons, via the mechanisms of long-term potentiation and depression (Karabanov et al., 2015). The durable effects of rTMS were initially noted via facilitation of motor evoked potentials with repeated stimulation of the primary motor cortex. Subsequently, it was found that rTMS delivered to the dlPFC improved mood in patients with depression (George et al., 1995; Pascual-Leone et al., 1996). Over the following
20 years, dozens of studies and several meta-analyses have established high-frequency left, low-frequency right and bilateral dIPFC-rTMS as superior to sham stimulation in the treatment of major depression (Berlim et al., 2013b,c; Berlim and Van Den Eynde, 2014; Gaynes et al., 2014). rTMS is now approved and used clinically as a treatment for medication-resistant depression in a variety of jurisdictions around the world (Lefaucheur et al., 2014; Milev et al., 2016; Perera et al., 2016).

rTMS is also showing promise as a treatment for a variety of other psychiatric disorders characterized by hypofunctioning of the SN. Supportive meta-analyses are now available for rTMS in treating SUD (Gorelick et al., 2014; Dunlop et al., 2016), PTSD (Berlim and Van Den Eynde, 2014), bipolar disorder (McGirr et al., 2016) and OCD (Berlim et al., 2013a; Trevizol et al., 2016).

The stimulation targets used in these studies typically correspond to frontal nodes of the SN. For example, the dlPFC region showing greatest efficacy in MDD has been reported to be a region anticorrelated to the sgCC, which also corresponds well to the SN's dIPFC node (Fox et al., 2012). Stimulation of the dACC and adjacent dmPFC has also been employed in MDD (Bakker et al., 2015; Kreuzer et al., 2015), and stimulation of medial SN nodes in the $\mathrm{dACC} / \mathrm{dmPFC}$ and adjacent pre-supplementary motor area have also shown promising effects in OCD (Mantovani et al., 2010; Dunlop et al., 2015a) and PTSD (Isserles et al., 2013). In healthy controls, there is evidence that rTMS of SN nodes such as the dlPFC or dmPFC/dACC can enhance or inhibit impulse control as measured on a delay-discounting task (Cho et al., 2010, 2015; Figner et al., 2010), and improve cognitive processing on executive functioning tasks (Esslinger et al., 2014), suggesting a generalized effect on cognitive control. Taking together these lines of evidence, recent reviews have suggested that the therapeutic effects of rTMS may be best understood not as "antidepressant" per se, but more generally as enhancement of cognitive control via improved SN integrity (Downar et al., 2016; Dunlop et al., 2016). This would account for its transdiagnostic efficacy across a range of psychiatric disorders involving cognitive control deficiency (McTeague et al., 2016).

The pertinent issue for the purposes of this review article is whether the therapeutic mechanisms of rTMS involve modulation of SN-CSTC loop circuits. Two questions thus arise: first, does rTMS of cortical targets cause neurophysiological changes in the CSTC loop for that target; and second, do these changes (if present) correlate to the behavioral and clinical effects of rTMS?

Regarding the first question, the neurophysiological mechanisms of rTMS are complex and still under investigation; accounts have been proposed at various levels of explanation from genetic and cell-molecular processes, to neurotransmitters and their receptors to synapses, to micro- and macro-level network connectivity changes (for reviews, see Noda et al., 2015). However, several convergent lines of evidence suggest that rTMS does indeed cause neurophysiological effects not merely at the stimulation site, but also throughout its 
CSTC loop circuit, targeting the entire loop in a precise, well-demarcated fashion. For example, PET studies using the D2 receptor tracer 11C-raclopride have shown that rTMS of the left primary motor cortex induces dopamine release specifically in the ipsilateral putamen, in a region corresponding to the known projection zone for corticostriatal projections from the primary motor cortex; no changes were seen in other striatal regions such as the caudate nucleus, NAcc or the contralateral putamen (Strafella et al., 2003). Another study by the same group, using the same tracer, demonstrated that rTMS of the left dIPFC induced dopamine release specifically in the head of the ipsilateral caudate nucleus, but not the NAcc, putamen or contralateral caudate nucleus (Strafella et al., 2001). A subsequent PET-rTMS study with a D2/D3 tracer demonstrated that rTMS of the dmPFC/dACC induced dopamine release in a circumscribed region of the dorsal putamen and underlying globus pallidus (Cho et al., 2015).

fMRI-rTMS studies support the premise that rTMS pulses activate not only the target region of cortex but also its associated striatal partner. fMRI-rTMS studies in healthy controls have directly demonstrated that rTMS pulses to the frontopolar cortex caused BOLD activations in both the frontal pole and in the ventral striatum; rTMS pulses to the dlPFC, in contrast, caused BOLD activations in both the dIPFC and the dorsal caudate nucleus (Hanlon et al., 2013, 2016). A session of inhibitory, continuous theta-burst stimulation to the frontopolar cortex reduced the BOLD response to individual pulses at the same target, with the effect seen prominently in ventral striatum (Hanlon et al., 2015). Thus, the available neuroimaging evidence from fMRI and PET studies suggests that rTMS directly activates not only the stimulation site but also the associated subcortical loop circuit, in a circumscribed fashion; these activations are accompanied by changes in dopamine neurotransmission in the subcortical projection zones of the stimulation target. rTMS of $\mathrm{SN}$ cortical targets (i.e., the dlPFC) appears to modulate activity in the subcortical components of the SN-CSTC loop circuit as well.

Regarding the second question of whether these changes in SN-CSTC loop circuit function are related to the behavioral and clinical effects of rTMS, several lines of evidence are now supportive. In a PET-rTMS study in healthy controls, an inhibitory form of rTMS (continuous thetaburst stimulation) delivered to the left dlPFC impaired performance on the Montreal Card Sorting Task, a set-shifting task requiring the cognitive control functions of the SN. This impairment was associated with reduced dopamine release in a circumscribed region of the head of the caudate nucleus (Ko et al., 2008). Likewise, in the previously mentioned PET study of dmPFC-rTMS (Cho et al., 2015), rTMS-induced changes in dopamine release in the globus pallidus showed a U-shaped relationship to change in impulsivity, as indexed on the delayed discounting task. Thus, the effects of rTMS on SN-CSTC loop circuits appear to translate into effects on cognitive control capacity.
There is also growing evidence that the therapeutic effects of rTMS in psychiatric illness may be mediated by changes in SN-CSTC loop circuit integrity. One supportive study used resting-state fMRI to examine CSTC functional connectivity in MDD patients who underwent a course of rTMS directed at the dmPFC/dACC. In this study, patients with low baseline functional connectivity from the dACC to the putamen (a region corresponding to that identified in the PET study of dmPFC-rTMS by Cho et al., 2015) and MD thalamus showed a greater degree of clinical improvement, and the degree of clinical improvement correlated to increases in functional connectivity from the stimulation target (dACC) and the MD thalamus (Salomons et al., 2014). The finding of dACC CSTC functional connectivity as both a predictor and correlate of clinical improvement was replicated in a follow-up study in ED patients undergoing dmPFC-rTMS for binge and purge behaviors (Dunlop et al., 2015a). In another follow-up study in patients with OCD (Dunlop et al., 2016), connectivity from the dACC to the head of the caudate nucleus and the MD thalamus was also a predictor and a correlate of clinical improvement; however, reductions rather than increases in connectivity within this CSTC loop were required for clinical improvement, paralleling the findings of Figee et al. (2014) in OCD patients undergoing DBS (as discussed in the previous section). These findings suggest that the therapeutic effects of rTMS in MDD, ED and OCD may be mediated by changes in the integrity of the SN-CSTC circuit. Future studies will be required to determine whether these findings also apply to the more commonly employed protocol of dlPFC- rather than dmPFC-rTMS.

\section{Transcranial Direct Current Stimulation (tDCS)}

tDCS is another non-invasive brain stimulation technique that uses scalp electrodes to deliver mild (1-2 mA) electrical currents to target brain regions, thereby modulating ongoing brain activity (Blumberger et al., 2015). The mechanisms of tDCS and related techniques such as transcranial alternating current stimulation (tACS) are still under investigation and debate (for reviews, see Nitsche et al., 2008; Tortella et al., 2015). However, from a clinical perspective, the technique is attractive for offering a favorable profile of safety, tolerability and low cost. For this reason, tDCS is under active investigation not only as a research tool but also as a potential therapeutic intervention in psychiatric illness (Tortella et al., 2015). The best-studied indication to date has been MDD, with several randomized controlled trials published; recent meta-analyses of these trials have found tDCS to be more effective than sham stimulation, with an effect size comparable to antidepressant medications (Meron et al., 2015; Brunoni et al., 2016).

Lateral cortical nodes of the SN are widely used as tDCS targets, both in basic science and clinical studies. One of the most common targets for tDCS in the literature to date has been the dIPFC, often operationalized as EEG sites F3 and F4 in the standard 10-20 montage. The F3 site has been shown to correspond fairly closely to the dlPFC 
node appearing in the SN (Mir-Moghtadaei et al., 2015), suggesting that tDCS of F3 and F4 is anatomically positioned to stimulate the SN. Research studies and clinical trials of tDCS have targeted the dlPFC due to its hypothesized central role in executive function and cognitive control (Kuo and Nitsche, 2012). tDCS targeting this region has been shown to improve performance across the domains of working memory (Fregni et al., 2005), impulsivity (Fecteau et al., 2007) and social cognition (Knoch et al., 2008). tACS has also been performed with electrodes placed bilaterally over the dIPFC (EEG sites F3 and F4) and its parietal counterpart sites (EEG sites P3 and P4), with one study reporting frequency-dependent enhancements of lucid dreaming during REM sleep (Voss et al., 2014).

The dlPFC has also been a tDCS target in the clinical treatment of substance use and ED. It is hypothesized that stimulating the dlPFC enhances top-down control over maladaptive eating behaviors and substance consumption, and suppresses cravings generated by dysfunctional reward circuitry (Fregni et al., 2005; Lapenta et al., 2014). Indeed, tDCS of the dlPFC has been shown to reduce cravings, consumption and behavioral impulsivity in long-term smokers (Rachid, 2016), which may represent effects in attentional salience or inhibition networks (Fregni et al., 2005; Lapenta et al., 2014).

An important question for the purposes of this review article is whether tDCS actually stimulates any of the deeper nodes of CSTC circuits, or whether its effects are confined to superficial cortical regions. A purely cortical mechanism of localized changes in synaptic plasticity is often postulated in the literature, given that the relatively weak electrical fields employed in tDCS may not penetrate beyond the cortex (Nitsche et al., 2008). However, several recent findings suggest that tDCS may indeed modulate neural activity in subcortical structures of the CSTC loop circuits. First, a study using resting-state $\mathrm{fMRI}$ found that $\mathrm{tDCS}$ of the primary motor cortex increased its functional connectivity to the thalamus, and additionally increased connectivity between the parietal cortex and the caudate nucleus (Polanía et al., 2012). Second, a study using the MRI-based perfusion technique of arterial spin labeling (ASL) demonstrated that anodal right and cathodal left dlPFC-tDCS caused decreases in resting perfusion of the head of the caudate nucleus as well as the medial and lateral orbitofrontal cortex (Weber et al., 2014). Finally, a recent study using magnetic resonance spectroscopy (MRS) has for the first time directly demonstrated that active but not sham dlPFC-tDCS (anodal left, cathodal right) increases levels of glutamate and glutamine in the striatum as well as $\mathrm{N}$-acetylaspartate in the dlPFC itself (Hone-Blanchet et al., 2015). Taken together, these findings suggest that tDCS can indeed modulate both neurotransmission and network connectivity patterns in the subcortical as well as the cortical components of CSTC loops when targeting the SN (i.e., via the dlPFC).

So far, little information is available that speaks to whether the therapeutic mechanisms of tDCS in psychiatric illness ensue via modulation of CSTC loop circuits, as appears to be the case for DBS and potentially for rTMS. As of this writing, the literature on the effects of tDCS on CSTC activity in general remains very limited (as reviewed in the preceding paragraph). Alternative possibilities are that the therapeutic effects of tDCS ensue purely through modulation of cortico-cortical network connectivity, or conceivably through local modulation of synaptic connections under the cortical stimulation site alone. Future studies of therapeutic tDCS in psychiatric illness will need to incorporate not only larger sample sizes, but also more detailed measures of cognitive control capacity, as well as neuroimaging observations before and after treatment, in order to identify the behavioral and neural correlates of clinical improvement. Such studies may help to determine whether tDCS of SN nodes enhances cognitive control, and if so, whether this enhancement ensues via modulation of the SN-CSTC loop circuits.

\section{UNRESOLVED QUESTIONS AND FUTURE DIRECTIONS FOR STUDY}

The emerging picture from the evidence reviewed in the previous three sections is that: (i) the SN-CSTC loop may play a critical role in the voluntary engagement of cognitive control; (ii) abnormalities of cognitive control are a common, pervasive and transdiagnostic feature of many psychiatric illnesses; (iii) these transdiagnostic deficits of cognitive control may arise from abnormalities of functioning within a specific member of the brain's many CSTC loops-namely, the CSTC loop serving the $\mathrm{SN}$; and (iv) emerging brain stimulation therapies such as DBS, rTMS and tDCS exert neurophysiological effects on targeted CSTC loop circuits, and these effects may be central to the mechanisms by which they alleviate psychiatric illness.

At the moment, this account of the CSTC loop through the $\mathrm{SN}$ must be considered preliminary, with many findings requiring further replication and study, and many questions still outstanding. In this final section, we review some potentially fruitful directions for further study.

\section{Particular Contributions of the SN-CSTC Loop to Voluntary Cognitive Control}

At present, it would be helpful to have a clearer understanding of the SN's role in cognitive control. Specifically, more information should be generated about the contributions of the $\mathrm{SN}$ to self-regulation of cognition, emotion and behavior in healthy brain function. The particular contributions of the SN should be distinguished from contributions of other functional networks involved in executive function, such as the neighboring frontoparietal networks known as dorsal attention or central executive networks. It would also be helpful to have a clearer understanding of the distinct roles of the $\mathrm{SN}$ vs. its immediately posterior counterpart comprising the posterior insula and mid-cingulate cortex. Finally, it is necessary to better understand how engagement of the subcortical projection sites of the SN (in the head of the caudate, globus pallidus, MD thalamus, and rostral substantia nigra) relates to the 
voluntary vs. passive engagement of cognitive control functions.

\section{Abnormalities of SN-CSTC Loop Integrity and Function in Psychiatric Illness}

In parallel with the previous theme, it would be helpful to have more detailed descriptions of the abnormalities present in the functional integrity, structural integrity and neurochemistry of the SN-CSTC loop circuit in the major categories of psychiatric illness. fMRI, ASL, DTI, VBM and PET studies will all be useful in this regard. The "intervention-probe" properties of DBS, rTMS, and tDCS may also yield useful information of a causal rather than correlational nature. It will be helpful to understand which psychiatric disorders share SN-CSTC dysregulation as a common feature, and whether there are disorders (for example, AN) where such pathology is relatively absent. Finally, as relatively few studies to date have explored the neural heterogeneity of illness among individuals with MDD, PTSD, OCD, SUD or other Axis I disorders (as defined by the DSM-IV), it will be helpful to understand whether SN-CSTC pathology is a pervasive feature across most individuals within each disease category, or whether instead only a certain sub-population of patients within MDD, PTSD, OCD or SUD show abnormalities of SN-CSTC functioning. The latter case, if true, may help to explain the problematic heterogeneity of outcomes currently seen with brain stimulation therapies, and may lead to methods for predicting which individuals are the best candidates for treatments targeting the SN-CSTC loop circuits. Better characterization of individual patients' pathology using the Research Domain Criteria (e.g., cognitive control, response selection and response inhibition) may be useful in this regard (Morris and Cuthbert, 2012).

\section{Contributions of Other CSTC Circuits to Psychiatric Illness}

Although mounting evidence suggests that SN-CSTC pathology may be a common feature of many psychiatric disorders, it is also clear that such pathology is far from the only, or even the most important, pathological feature in many individuals. For example, pathology of the "reward" or "incentive" CSTC loop from the NAcc to the vmPFC and frontal pole increasingly appears to be important across a variety of disorders. Aside from the well-established example of SUD, pathology of this loop may be important in OCD, MDD (particularly for the symptom of anhedonia), schizophrenia, and other psychiatric disorders (as noted throughout the previous three sections of this review). In addition, interactions (such as mutual inhibition) between the activity of the dorsal striatal-SN loop and the ventral striatal reward loop may be important for understanding psychiatric pathology and its heterogeneity; such interactions between dorsal and ventral CSTC loops are an increasingly prevalent theme of study in recent work combining neuroimaging and neurostimulation in psychiatric illness (for example, Liston et al., 2014; Hanlon et al., 2016).

\section{Effects of Brain Stimulation Techniques on SN-CSTC Function}

The available literature to date provides mounting evidence that brain stimulation treatments are capable of modulating the activity of CSTC circuits, and that these effects may be central to the therapeutic properties of such treatments. Nonetheless, further evidence is needed to demonstrate and characterize the effects of brain stimulation treatments on CSTC function-to some extent for DBS, more so for rTMS, and especially so for tDCS and tACS. As stated above, a variety of techniques will be useful in this regard: in addition to fMRI studies of network integrity, PET and MRS-and potentially voltammetry-will be useful for characterizing neurochemical effects, while electrophysiological recordings (noninvasive MEG and EEG, and invasive intracortical recordings when available) will be essential in assessing the direct effects of stimulation on neural activity. Much of the available literature to date has focused on motor CSTC loops rather than other prefrontal loops. However, in light of the increasing popularity of brain stimulation in treating psychiatric illness, more attention is due to the SN-CSTC loop in future study.

\section{Optimizing Brain Stimulation Protocols for Modulating SN-CSTC Function}

The optimal parameters for therapeutic DBS, rTMS and tDCS/tACS in psychiatric illness are still being refined, and in many cases are entirely unknown. To date, very few studies have sought to optimize the frequency, intensity, protocol, inter-session interval or even dose (i.e., session number) for therapeutic brain stimulation as a primary aim. The assessment of relative efficacy in most cases has been empirical, based on clinical measures (e.g., standardized symptom scales) that may not be well suited to capturing the nuanced effects of therapeutic brain stimulation. For example, after 20 years of clinical trials of rTMS in MDD, there is a wealth of evidence about crude response and remission rates for this heterogeneous population, but very little evidence about which types of patients respond best to treatment, or whether responders are characterized specifically by deficiencies of cognitive control rather than conventional mood symptoms per se, as proposed in this review. A more nuanced outcome measure (behavioral, cognitive or neurophysiological) may be helpful in providing a benchmark for optimizing the parameters of stimulation. For example, if the mechanism of effect for dlPFC- or dmPFC-rTMS does indeed depend upon enhancements in deficient cognitive control and SN-CSTC loop circuit integrity, then obtaining markers of cognitive control and SN integrity will be essential to any parameter-optimization study. Candidate markers might include behavioral measures such as performance on flanker or delayed discounting tasks, electrophysiological measures such as coupling between theta and gamma oscillations, or neuroimaging markers such as D2 receptor occupancy on PET or SN-striatal-thalamic functional connectivity on fMRI. Future studies of therapeutic brain stimulation may need to make use of such markers in order to make progress in exploring the many dimensions of stimulation parameters that are still 
awaiting optimization. Future studies involving fMRI should also consider the false-positive rate of fMRI in the application of past research, and study design and processing (Eklund et al., 2016); this point highlights the importance of replication studies and meta-analyses in developing new rTMS targets and in interpreting the effects of rTMS on brain function.

\section{CONCLUSION}

Converging evidence suggests that, of the brain's many CSTC loop circuits, the specific circuit serving the SN may be of particular relevance to cognitive control, and of transdiagnostic relevance to psychiatric pathology. This proposal, if supported by future work, is of more than purely academic interest. With the emergence of anatomically selective brain stimulation technologies as therapeutic tools, it is becoming possible to target specific CSTC loop circuits of the brain in an increasingly precise manner, and to modulate their activity in a variety of ways. Therapeutic brain stimulation of the SN-CSTC loop circuit may constitute a method for directly targeting the underlying pathophysiology of several types of psychiatric illness, or at least a subpopulation of individuals within these categories of illness. Stimulation targeting other CSTC loops, such as those through the ventral striatum, may further expand the range of disorders and individuals whose illness is amenable to

\section{REFERENCES}

Abraham, A., Beudt, S., Ott, D. V. M., and Yves von Cramon, D. (2012). Creative cognition and the brain: dissociations between frontal, parietal-temporal and basal ganglia groups. Brain Res. 1482, 55-70. doi: 10.1016/j.brainres.2012. 09.007

Admon, R., Nickerson, L. D., Dillon, D. G., Holmes, A. J., Bogdan, R., Kumar, P., et al. (2015). Dissociable cortico-striatal connectivity abnormalities in major depression in response to monetary gains and penalties. Psychol. Med. 45, 121-131. doi: 10.1017/S0033291714001123

Alexander, G. E., DeLong, M. R., and Strick, P. L. (1986). Parallel organization of functionally segregated circuits linking basal ganglia and cortex. Annu. Rev. Neurosci. 9, 357-381. doi: 10.1146/annurev.neuro.9.1.357

American Psychiatric Association. (1994). Diagnostic and Statistical Manual of Mental Disorders. 4th Edn. Washington, DC: American Psychiatric Association.

American Psychiatric Association. (2013). DSM-V, Diagnostic and Statistical Manual of Mental Disorders. 5th Edn. Arlington, VA: American Psychiatric Publishing Inc.

Amianto, F., D’Agata, F., Lavagnino, L., Caroppo, P., Abbate-Daga, G., Righi, D., et al. (2013). Intrinsic connectivity networks within cerebellum and beyond in eating disorders. Cerebellum 12, 623-631. doi: 10.1007/s12311-0130471-1

Anders, S., Lotze, M., Erb, M., Grodd, W., and Birbaumer, N. (2004). Brain activity underlying emotional valence and arousal: a response-related fMRI study. Hum. Brain Mapp. 23, 200-209. doi: 10.1002/hbm.20048

Anticevic, A., Hu, S., Zhang, S., Savic, A., Billingslea, E., Wasylink, S., et al. (2014). Global resting-state functional magnetic resonance imaging analysis identifies frontal cortex, striatal and cerebellar dysconnectivity in obsessivecompulsive disorder. Biol. Psychiatry 75, 595-605. doi: 10.1016/j.biopsych. 2013.10.021

Atmaca, M., Mermi, O., Yildirim, H., and Gurok, M. G. (2016). Orbito-frontal cortex and thalamus volumes in obsessive-compulsive disorder before and after pharmacotherapy. Brain Imaging Behav. 10, 669-674. doi: 10.1007/s11682-0159426-0 brain stimulation treatments. With a better understanding of CSTC function in health and psychiatric disease, it may become possible to tailor the target and parameters of stimulation to the individual, depending on the underlying pathology. This individualized, brain-based approach to psychiatric treatment would constitute an important step forward in addressing the daunting prevalence and burden of mental illness around the world.

\section{AUTHOR CONTRIBUTIONS}

SKP, KD and JD wrote and edited the manuscript and figures.

\section{FUNDING}

SKP has received funding from the University of Toronto Ontario Graduate Fund. KD has received funding from the Canadian Institute for Health Research (CIHR) Vanier Scholarship. JD has received research support from CIHR, NIH, the Klarman Family Foundation, the Buchan Family Foundation and the Toronto General and Western Hospital Foundation. He has also received a travel stipend from Lundbeck and from ANT Neuro, and in-kind equipment support for an investigator-initiated study from Tonika/Magventure.
Averbeck, B. B., Lehman, J., Jacobson, M., and Haber, S. N. (2014). Estimates of projection overlap and zones of convergence within frontal-striatal circuits. J. Neurosci. 34, 9497-9505. doi: 10.1523/JNEUROSCI.5806-12.2014

Bailer, U. F., Frank, G. K., Price, J. C., Meltzer, C. C., Becker, C., Mathis, C. A., et al. (2013). Interaction between serotonin transporter and dopamine D2/D3 receptor radioligand measures is associated with harm avoidant symptoms in anorexia and bulimia nervosa. Psychiatry Res. 211, 160-168. doi: 10.1016/j.pscychresns.2012.06.010

Bailer, U. F., Price, J. C., Meltzer, C. C., Wagner, A., Mathis, C. A., Gamst, A., et al. (2016). Dopaminergic activity and altered reward modulation in anorexia nervosa-insight from multimodal imaging. Int. J. Eat. Disord. doi: 10.1002/eat. 22638 [Epub ahead of print].

Bakker, N., Shahab, S., Giacobbe, P., Blumberger, D. M., Daskalakis, Z. J., Kennedy, S. H., et al. (2015). RTMS of the dorsomedial prefrontal cortex for major depression: safety, tolerability, effectiveness and outcome predictors for $10 \mathrm{~Hz}$ versus intermittent theta-burst stimulation. Brain Stimul. 8, 208-215. doi: 10.1016/j.brs.2014.11.002

Benazzi, F. (2006). Various forms of depression. Dialogues Clin. Neurosci. 8, 151-161.

Berlim, M. T., Neufeld, N. H., and Van den Eynde, F. (2013a). Repetitive transcranial magnetic stimulation (rTMS) for obsessive-compulsive disorder (OCD): an exploratory meta-analysis of randomized and sham-controlled trials. J. Psychiatr. Res. 47, 999-1006. doi: 10.1016/j.jpsychires.2013. 03.022

Berlim, M. T., Van den Eynde, F., and Jeff Daskalakis, Z. (2013b). Clinically meaningful efficacy and acceptability of low-frequency repetitive transcranial magnetic stimulation (rTMS) for treating primary major depression: a meta-analysis of randomized, double-blind and sham-controlled trials. Neuropsychopharmacology 38, 543-551. doi: 10.1038/npp.2012.237

Berlim, M. T., van den Eynde, F., Tovar-Perdomo, S., and Daskalakis, Z. J. (2013c). Response, remission and drop-out rates following high-frequency repetitive transcranial magnetic stimulation (rTMS) for treating major depression: a systematic review and meta-analysis of randomized, double-blind and sham- controlled trials. Psychol. Med. 44, 225-239. doi: 10.1017/S00332917130 00512 
Berlim, M. T., and Van Den Eynde, F. (2014). Repetitive transcranial magnetic stimulation over the dorsolateral prefrontal cortex for treating posttraumatic stress disorder: an exploratory meta-analysis of randomized, double-blind and sham-controlled trials. Can. J. Psychiatry 59, 487-496.

Berner, L. A., and Marsh, R. (2014). Frontostriatal circuits and the development of bulimia nervosa. Front. Behav. Neurosci. 8:395. doi: 10.3389/fnbeh.2014.00395

Bernstein, G. A., Mueller, B. A., Schreiner, M. W., Campbell, S. M., Regan, E. K., Nelson, P. M., et al. (2016). Abnormal striatal resting-state functional connectivity in adolescents with obsessive-compulsive disorder. Psychiatry Res. 247, 49-56. doi: 10.1016/j.pscychresns.2015.11.002

Berridge, K. C. (1996). Food reward: brain substrates of wanting and liking. Neurosci. Biobehav. Rev. 20, 1-25. doi: 10.1016/0149-7634(95) 00033-b

Bewernick, B. H., Hurlemann, R., Matusch, A., Kayser, S., Grubert, C., Hadrysiewicz, B., et al. (2010). Nucleus accumbens deep brain stimulation decreases ratings of depression and anxiety in treatment-resistant depression. Biol. Psychiatry 67, 110-116. doi: 10.1016/j.biopsych.2009.09.013

Biezonski, D., Cha, J., Steinglass, J., and Posner, J. (2015). Evidence for thalamocortical circuit abnormalities and associated cognitive dysfunctions in underweight individuals with anorexia nervosa. Neuropsychopharmacology 41, 1560-1568. doi: 10.1038/npp.2015.314

Bischoff-Grethe, A., McCurdy, D., Grenesko-Stevens, E., Irvine, L. E., Wagner, A., Yau, W. Y., et al. (2013). Altered brain response to reward and punishment in adolescents with Anorexia nervosa. Psychiatry Res. 214, 331-340. doi: 10.1016/j. pscychresns.2013.07.004

Biswal, B., Yetkin, F. Z., Haughton, V. M., and Hyde, J. S. (1995). Functional connectivity in the motor cortex of resting human brain using echo-planar MRI. Magn. Reson. Med. 34, 537-541. doi: 10.1002/mrm.19103 40409

Blumberger, D. M., Hsu, J. H., and Daskalakis, Z. J. (2015). A review of brain stimulation treatments for late-life depression. Curr. Treat. Options Psychiatry 2, 413-421. doi: 10.1007/s40501-015-0059-0

Bora, E., Harrison, B. J., Davey, C. G., Yücel, M., and Pantelis, C. (2012). Meta-analysis of volumetric abnormalities in cortico-striatal-pallidal-thalamic circuits in major depressive disorder. Psychol. Med. 42, 671-681. doi: 10. $1017 /$ s0033291711001668

Botvinick, M. M., Cohen, J. D., and Carter, C. S. (2004). Conflict monitoring and anterior cingulate cortex: an update. Trends Cogn. Sci. 8, 539-546. doi: 10. 1016/j.tics.2004.10.003

Bourne, S. K., Eckhardt, C. A., Sheth, S. A., and Eskandar, E. N. (2012). Mechanisms of deep brain stimulation for obsessive compulsive disorder: effects upon cells and circuits. Front. Integr. Neurosci. 6:29. doi: 10.3389/fnint. 2012.00029

Bressler, S. L., and Menon, V. (2010). Large-scale brain networks in cognition: emerging methods and principles. Trends Cogn. Sci. 14, 277-290. doi: 10.1016/j. tics.2010.04.004

Broft, A., Slifstein, M., Osborne, J., Kothari, P., Morim, S., Shingleton, R., et al. (2015). Striatal dopamine type 2 receptor availability in anorexia nervosa. Psychiatry Res. 233, 380-387. doi: 10.1016/j.pscychresns.2015.06.013

Brooks, S. J., O’Daly, O. G., Uher, R., Friederich, H. C., Giampietro, V., Brammer, M., et al. (2011). Differential neural responses to food images in women with bulimia versus anorexia nervosa. PLoS One 6:e22259. doi: 10. 1371/journal.pone.0022259

Brunoni, A. R., Moffa, A. H., Fregni, F., Palm, U., Padberg, F., Blumberger, D. M., et al. (2016). Transcranial direct current stimulation for acute major depressive episodes: meta-analysis of individual patient data. Br. J. Psychiatry 208, 522-531. doi: 10.1192/bjp.bp.115.164715

Chantiluke, K., Halari, R., Simic, M., Pariante, C. M., Papadopoulos, A., Giampietro, V., et al. (2012). Fronto-striato-cerebellar dysregulation in adolescents with depression during motivated attention. Biol. Psychiatry 71, 59-67. doi: 10.1016/j.biopsych.2011.09.005

Chen, Q., Chen, X., He, X., Wang, L., Wang, K., and Qiu, B. (2016). Aberrant structural and functional connectivity in the salience network and central executive network circuit in schizophrenia. Neurosci. Lett. 627, 178-184. doi: 10.1016/j.neulet.2016.05.035

Chen, X. L., Xiong, Y. Y., Xu, G. L., and Liu, X. F. (2013). Deep brain stimulation. Interv. Neurol. 1, 200-212. doi: 10.1159/000353121

Cho, S. S., Ko, J. H., Pellecchia, G., Van Eimeren, T., Cilia, R., and Strafella, A. P. (2010). Continuous theta burst stimulation of right dorsolateral prefrontal cortex induces changes in impulsivity level. Brain Stimul. 3, 170-176. doi: 10. 1016/j.brs.2009.10.002

Cho, S. S., Koshimori, Y., Aminian, K., Obeso, I., Rusjan, P., Lang, A. E., et al. (2015). Investing in the future: stimulation of the medial prefrontal cortex reduces discounting of delayed rewards. Neuropsychopharmacology 40, 546-553. doi: 10.1038/npp.2014.211

Choi, E. Y., Tanimura, Y., Vage, P. R., Yates, E. H., and Haber, S. N. (2016). Convergence of prefrontal and parietal anatomical projections in a connectional hub in the striatum. Neuroimage doi: 10.1016/j.neuroimage.2016. 09.037 [Epub ahead of print].

Choi, E. Y., Yeo, B. T. T., and Buckner, R. L. (2012). The organization of the human striatum estimated by intrinsic functional connectivity. J. Neurophysiol. 108, 2242-2263. doi: 10.1152/jn.00270.2012

Cisler, J. M., Steele, J. S., Lenow, J. K., Smitherman, S., Everett, B., Messias, E., et al. (2014). Functional reorganization of neural networks during repeated exposure to the traumatic memory in posttraumatic stress disorder: an exploratory fMRI study. J. Psychiatr. Res. 48, 47-55. doi: 10.1016/j.jpsychires.2013. 09.013

Corbetta, M., Kincade, J. M., Ollinger, J. M., McAvoy, M. P., and Shulman, G. L. (2000). Voluntary orienting is dissociated from target detection in human posterior parietal cortex. Nat. Neurosci. 3, 292-297. doi: 10.1038/73009

Coutinho, J., Ramos, A. F., Maia, L., Castro, L., Conceição, E., Geliebter, A., et al. (2015). Volumetric alterations in the nucleus accumbens and caudate nucleus in bulimia nervosa: a structural magnetic resonance imaging study. Int. J. Eat. Disord. 48, 206-214. doi: 10.1002/eat.22273

Cowdrey, F. A., Park, R. J., Harmer, C. J., and McCabe, C. (2011). Increased neural processing of rewarding and aversive food stimuli in recovered anorexia nervosa. Biol. Psychiatry 70, 736-743. doi: 10.1016/j.biopsych.2011. 05.028

Craig, A. D. (2003). Interoception: the sense of the physiological condition of the body. Curr. Opin. Neurobiol. 13, 500-505. doi: 10.1016/s0959-4388(03) 00090-4

Craig, A. D. (2009). How do you feel - now? The anterior insula and human awareness. Nat. Rev. Neurosci. 10, 59-70. doi: 10.1038/nrn2555

Dager, A. D., Anderson, B. M., Rosen, R., Khadka, S., Sawyer, B., JiantonioKelly, R. E., et al. (2014). Functional magnetic resonance imaging (fMRI) response to alcohol pictures predicts subsequent transition to heavy drinking in college students. Addiction 109, 585-595. doi: 10.1111/add. 12437

Davis, B., and Hasson, U. (2016). Predictability of what or where reduces brain activity, but a bottleneck occurs when both are predictable. Neuroimage doi: 10 1016/j.neuroimage.2016.06.001 [Epub ahead of print].

Decker, J. H., Figner, B., and Steinglass, J. E. (2015). On weight and waiting: delay discounting in anorexia nervosa pretreatment and posttreatment. Biol. Psychiatry 78, 606-614. doi: 10.1016/j.biopsych.2014. 12.016

Delaveau, P., Jabourian, M., Lemogne, C., Guionnet, S., Bergouignan, L., and Fossati, P. (2011). Brain effects of antidepressants in major depression: a meta-analysis of emotional processing studies. J. Affect. Disord. 130, 66-74. doi: 10.1016/j.jad.2010.09.032

Delevich, K., Tucciarone, J., Huang, Z. J., and Li, B. (2015). The mediodorsal thalamus drives feedforward inhibition in the anterior cingulate cortex via parvalbumin interneurons. J. Neurosci. 35, 5743-5753. doi: 10 . 1523/JNEUROSCI.4565-14.2015

Di Filippo, M., Picconi, B., Tantucci, M., Ghiglieri, V., Bagetta, V., Sgobio, C., et al. (2009). Short-term and long-term plasticity at corticostriatal synapses: implications for learning and memory. Behav. Brain Res. 199, 108-118. doi: 10. 1016/j.bbr.2008.09.025

Dosenbach, N. U., Fair, D. A., Cohen, A. L., Schlaggar, B. L., and Petersen, S. E. (2008). A dual-networks architecture of top-down control. Trends Cogn. Sci. 12, 99-105. doi: 10.1016/j.tics.2008.01.001

Dougherty, D. D., Baer, L., Cosgrove, G. R., Cassem, E. H., Price, B. H., Nierenberg, A. A., et al. (2002). Prospective long-term follow-up of 44 patients who received cingulotomy for treatment-refractory obsessivecompulsive disorder. Am. J. Psychiatry 159, 269-275. doi: 10.1176/appi.ajp. 159.2.269

Downar, J., Blumberger, D. M., and Daskalakis, Z. J. (2016). The neural crossroads of psychiatric illness: an emerging target for brain stimulation. Trends Cogn. Sci. 20, 107-120. doi: 10.1016/j.tics.2015.10.007 
Downar, J., Crawley, A. P., Mikulis, D. J., and Davis, K. D. (2000). A multimodal cortical network for the detection of changes in the sensory environment. Nat. Neurosci. 3, 277-283. doi: 10.1038/72991

Downar, J., Crawley, A. P., Mikulis, D. J., and Davis, K. D. (2001). The effect of task relevance on the cortical response to changes in visual and auditory stimuli: an event-related fMRI study. Neuroimage 14, 1256-1267. doi: 10.1006/nimg.2001. 0946

Downar, J., Crawley, A. P., Mikulis, D. J., and Davis, K. D. (2002). A cortical network sensitive to stimulus salience in a neutral behavioral context across multiple sensory modalities. J. Neurophysiol. 87, 615-620.

Duarte, J. M., Lanz, B., and Gruetter, R. (2011). Compartmentalized cerebral metabolism of $\left[1,6-{ }^{13} \mathrm{C}\right]$ glucose determined by in vivo ${ }^{13} \mathrm{C}$ NMR spectroscopy at 14.1 T. Front. Neuroenergetics 3:3. doi: 10.3389/fnene.2011.00003

Dunlop, K., Hanlon, C. A., and Downar, J. (2015a). Non-invasive brain stimulation treatments for addiction and major depression. Ann. N Y Acad. Sci. doi: 10. 1111/nyas.12985 [Epub ahead of print].

Dunlop, K., Woodside, B., Lam, E., Olmsted, M., Colton, P., Giacobbe, P., et al. (2015b). Increases in frontostriatal connectivity are associated with response to dorsomedial repetitive transcranial magnetic stimulation in refractory binge/purge behaviors. Neuroimage Clin. 8, 611-618. doi: 10.1016/j.nicl.2015. 06.008

Dunlop, K., Woodside, B., Olmsted, M., Colton, P., Giacobbe, P., and Downar, J. (2016). Reductions in cortico-striatal hyperconnectivity accompany successful treatment of obsessive-compulsive disorder with dorsomedial prefrontal rTMS. Neuropsychopharmacology 41, 1395-1403. doi: 10.1038/npp. 2015.292

Dutta, A., McKie, S., and Deakin, J. F. (2014). Resting state networks in major depressive disorder. Psychiatry Res. 224, 139-151. doi: 10.1016/j.pscychresns. 2014.10.003

Ehrlich, S., Lord, A. R., Geisler, D., Borchardt, V., Boehm, I., Seidel, M., et al. (2015). Reduced functional connectivity in the thalamo-insular subnetwork in patients with acute anorexia nervosa. Hum. Brain Mapp. 36, 1772-1781. doi: 10. 1002/hbm. 22736

Eklund, A., Nichols, T. E., and Knutsson, H. (2016). Cluster failure: why fMRI inferences for spatial extent have inflated false-positive rates. Proc. Natl. Acad. Sci. U S A 113, 7900-7905. doi: 10.1073/pnas.1602413113

Ersche, K. D., Barnes, A., Jones, P. S., Morein-Zamir, S., Robbins, T. W., and Bullmore, E. T. (2011). Abnormal structure of frontostriatal brain systems is associated with aspects of impulsivity and compulsivity in cocaine dependence. Brain 134, 2013-2024. doi: 10.1093/brain/awr138

Esslinger, C., Schüler, N., Sauer, C., Gass, D., Mier, D., Braun, U., et al. (2014). Induction and quantification of prefrontal cortical network plasticity using $5 \mathrm{~Hz}$ rTMS and fMRI. Hum. Brain Mapp. 35, 140-151. doi: 10.1002/hbm. 22165

Falconer, E., Allen, A., Felmingham, K. L., Williams, L. M., and Bryant, R. A. (2013). Inhibitory neural activity predicts response to cognitive-behavioral therapy for posttraumatic stress disorder. J. Clin. Psychiatry 74, 895-901. doi: 10.4088/JCP.12m08020

Fasano, A., and Lozano, A. M. (2015). Deep brain stimulation for movement disorders: 2015 and beyond. Curr. Opin. Neurol. 28, 423-436. doi: 10. 1097/WCO.0000000000000226

Fecteau, S., Pascual-Leone, A., Zald, D. H., Liguori, P., Théoret, H., Boggio, P. S., et al. (2007). Activation of prefrontal cortex by transcranial direct current stimulation reduces appetite for risk during ambiguous decision making. J. Neurosci. 27, 6212-6218. doi: 10.1523/JNEUROSCI.0314-07.2007

Feinstein, J. S., Stein, M. B., and Paulus, M. P. (2006). Anterior insula reactivity during certain decisions is associated with neuroticism. Soc. Cogn. Affect. Neurosci. 1, 136-142. doi: 10.1093/scan/ns1016

Feng, D., Yuan, K., Li, Y., Cai, C., Yin, J., Bi, Y., et al. (2016). Intra-regional and inter-regional abnormalities and cognitive control deficits in young adult smokers. Brain Imaging Behav. 10, 506-516. doi: 10.1007/s11682-015-9427-z

Ferrari, A. J., Charlson, F. J., Norman, R. E., Patten, S. B., Freedman, G., Murray, C. J. L., et al. (2013). Burden of depressive disorders by country, sex, age and year: findings from the global burden of disease study 2010. PLoS Med. 10:e1001547. doi: 10.1371/journal.pmed.1001547

Figee, M., Luigjes, J., Smolders, R., Valencia-Alfonso, C. E., van Wingen, G., de Kwaasteniet, B., et al. (2014). Deep brain stimulation restores frontostriatal network activity in obsessive- compulsive disorder. Nat. Neurosci. 16, 386-387. doi: $10.1038 / \mathrm{nn} .3344$
Figner, B., Knoch, D., Johnson, E. J., Krosch, A. R., Lisanby, S. H., Fehr, E., et al. (2010). Lateral prefrontal cortex and self-control in intertemporal choice. Nat. Neurosci. 13, 538-539. doi: 10.1038/nn.2516

Fitzgerald, P. B., Laird, A. R., Maller, J., and Daskalakis, Z. J. (2008). A meta-analytic study of changes in brain activation in depression. Hum. Brain Mapp. 29, 683-695. doi: 10.1002/hbm.20426

Foerde, K., Poldrack, R. A., Khan, B. J., Sabb, F. W., Bookheimer, S. Y., Bilder, R. M., et al. (2008). Selective corticostriatal dysfunction in schizophrenia: examination of motor and cognitive skill learning. Neuropsychology 22, 100-109. doi: 10.1037/0894-4105.22.1.100

Forbes, E. E., Rodriguez, E. E., Musselman, S., and Narendran, R. (2014). Prefrontal response and frontostriatal functional connectivity to monetary reward in abstinent alcohol-dependent young adults. PLoS One 9:e94640. doi: 10.1371/journal.pone.0094640

Fox, M. D., Buckner, R. L., White, M. P., Greicius, M. D., and Pascual-Leone, A. (2012). Efficacy of transcranial magnetic stimulation targets for depression is related to intrinsic functional connectivity with the subgenual cingulate. Biol. Psychiatry 72, 595-603. doi: 10.1016/j.biopsych.2012.04.028

Frank, G. K., Reynolds, J. R., Shott, M. E., Jappe, L., Yang, T. T., Tregellas, J. R., et al. (2012). Anorexia nervosa and obesity are associated with opposite brain reward response. Neuropsychopharmacology 37, 2031-2046. doi: 10.1038/npp. 2012.51

Frank, G. K., Shott, M. E., Hagman, J. O., and Mittal, V. A. (2013). Alterations in brain structures related to taste reward circuitry in ill and recovered anorexia nervosa and in bulimia nervosa. Am. J. Psychiatry 170, 1152-1160. doi: 10. 1176/appi.ajp.2013.12101294

Frank, G. K., Shott, M. E., Riederer, J., and Pryor, T. L. (2016). Altered structural and effective connectivity in anorexia and bulimia nervosa in circuits that regulate energy and reward homeostasis. Transl. Psychiatry 6:e932. doi: 10. 1038/tp.2016.199

Fregni, F., Boggio, P. S., Nitsche, M., Bermpohl, F., Antal, A., Feredoes, E., et al. (2005). Anodal transcranial direct current stimulation of prefrontal cortex enhances working memory. Exp. Brain Res. 166, 23-30. doi: 10.1007/s00221005-2334-6

Friederich, H., Walther, S., Bendszus, M., Biller, A., Thomann, P., Zeigermann, S., et al. (2012). NeuroImage Grey matter abnormalities within corticolimbic-striatal circuits in acute and weight-restored anorexia nervosa patients. Neuroimage 59, 1106-1113. doi: 10.1016/j.neuroimage.2011. 09.042

Frieling, H., Fischer, J., Wilhelm, J., Engelhorn, T., Bleich, S., Hillemacher, T., et al. (2012). Microstructural abnormalities of the posterior thalamic radiation and the mediodorsal thalamic nuclei in females with anorexia nervosa-a voxel based diffusion tensor imaging (DTI) study. J. Psychiatr. Res. 46, 1237-1242. doi: 10.1016/j.jpsychires.2012.06.005

Fu, C. H., Steiner, H., and Costafreda, S. G. (2013). Predictive neural biomarkers of clinical response in depression: a meta-analysis of functional and structural neuroimaging studies of pharmacological and psychological therapies. Neurobiol. Dis. 52, 75-83. doi: 10.1016/j.nbd.2012.05.008

Furman, D. J., Hamilton, J. P., and Gotlib, I. H. (2011). Frontostriatal functional connectivity in major depressive disorder. Biol. Mood Anxiety Disord. 1:11. doi: 10.1186/2045-5380-1-11

Gaynes, B. N., Lloyd, S. W., Lux, L., Gartlehner, G., Hansen, R. A., Brode, S., et al. (2014). Repetitive transcranial magnetic stimulation for treatment-resistant depression: a systematic review and meta-analysis. J. Clin. Psychiatry 75, 477-489; quiz 489. doi: 10.4088/JCP.13r08815

Geisler, D., Borchardt, V., Lord, A. R., Boehm, I., Ritschel, F., Zwipp, J., et al. (2016). Abnormal functional global and local brain connectivity in female patients with anorexia nervosa. J. Psychiatry Neurosci. 41, 6-15. doi: 10. 1503/jpn. 140310

George, M. S., Wassermann, E. M., Williams, W. A., Callahan, A., Ketter, T. A., Basser, P., et al. (1995). Daily repetitive transcranial magnetic stimulation (rTMS) improves mood in depression. Neuroreport 6, 1853-1856. doi: 10. 1097/00001756-199510020-00008

Giacobbe, P., Mayberg, H. S., and Lozano, A. M. (2009). Treatment resistant depression as a failure of brain homeostatic mechanisms: implications for deep brain stimulation. Exp. Neurol. 219, 44-52. doi: 10.1016/j.expneurol.2009. 04.028

Gilbert, A. R., Keshavan, M. S., Diwadkar, V., Nutche, J., Macmaster, F., Easter, P. C., et al. (2008). Gray matter differences between pediatric obsessive- 
compulsive disorder patients and high-risk siblings: a preliminary voxelbased morphometry study. Neurosci. Lett. 1, 45-50. doi: 10.1016/j.neulet.2008. 02.011

Goldstein, R. Z., Craig, A. D., Bechara, A., Garavan, H., Childress, A. R., Paulus, M. P., et al. (2009). The neurocircuitry of impaired insight in drug addiction. Trends Cogn. Sci. 13, 372-380. doi: 10.1016/j.tics.2009. 06.004

Goodkind, M., Eickhoff, S. B., Oathes, D. J., Jiang, Y., Chang, A., Joneshagata, L. B., et al. (2015). Identification of a common neurobiological substrate for mental illness. JAMA Psychiatry 72, 305-315. doi: 10.1001/jamapsychiatry. 2014.2206

Gorelick, D. A., Zangen, A., and George, M. S. (2014). Transcranial magnetic stimulation in the treatment of substance addiction. Ann. N Y Acad. Sci. 1327, 79-93. doi: 10.1111/nyas.12479

Graybiel, A. M., and Rauch, S. L. (2000). Toward a neurobiology of obsessivecompulsive disorder. Neuron 28, 343-347. doi: 10.1016/S0896-6273(00) 00113-6

Haber, S. N. (2016). Corticostriatal circuitry. Dialogues Clin. Neurosci. 18, 7-21.

Hagan, C. C., Graham, J. M., Tait, R., Widmer, B., van Nieuwenhuizen, A. O., Ooi, C., et al. (2015). Adolescents with current major depressive disorder show dissimilar patterns of age-related differences in ACC and thalamus. Neuroimage Clin. 7, 391-399. doi: 10.1016/j.nicl.2014.12.019

Hahn, A., Haeusler, D., Kraus, C., Höflich, A. S., Kranz, G. S., Baldinger, P., et al. (2014). Attenuated serotonin transporter association between dorsal raphe and ventral striatum in major depression. Hum. Brain Mapp. 35, 3857-3866. doi: $10.1002 / \mathrm{hbm} .22442$

Hallett, M. (2007). Transcranial magnetic stimulation: a primer. Neuron 55, 187-199. doi: 10.1016/j.neuron.2007.06.026

Ham, T., Leff, A., de Boissezon, X., Joffe, A., and Sharp, D. J. (2013). Cognitive control and the salience network: an investigation of error processing and effective connectivity. J. Neurosci. 33, 7091-7098. doi: 10.1523/JNEUROSCI. 4692-12.2013

Hanlon, C. A., Canterberry, M., Taylor, J. J., DeVries, W., Li, X., Brown, T. R., et al. (2013). Probing the frontostriatal loops involved in executive and limbic processing via interleaved TMS and functional MRI at two prefrontal locations: a pilot study. PLoS One 7:e67917. doi: 10.1371/journal.pone.00 67917

Hanlon, C. A., Dowdle, L. T., Austelle, C. W., DeVries, W., Mithoefer, O., Badran, B. W., et al. (2015). What goes up, can come down: novel brain stimulation paradigms may attenuate craving and craving-related neural circuitry in substance dependent individuals. Brain Res. 1628, 199-209. doi: 10. 1016/j.brainres.2015.02.053

Hanlon, C. A., Dowdle, L. T., Moss, H., Canterberry, M., and George, M. S. (2016). Mobilization of medial and lateral frontal-striatal circuits in cocaine users and controls: an interleaved TMS/BOLD functional connectivity study. Neuropsychopharmacology 41, 3032-3041. doi: 10.1038/npp. 2016.114

Hayes, D. J., Lipsman, N., Chen, D. Q., Woodside, D. B., Davis, K. D., Lozano, A. M., et al. (2015). Subcallosal cingulate connectivity in anorexia nervosa patients differs from healthy controls: a multi-tensor tractography study. Brain Stimul. 8, 758-768. doi: 10.1016/j.brs.2015.03.005

Herringa, R., Phillips, M., Almeida, J., Insana, S., and Germain, A. (2012). Posttraumatic stress symptoms correlate with smaller subgenual cingulate, caudate and insula volumes in unmedicated combat veterans. Psychiatry Res. 203, 139-145. doi: 10.1016/j.pscychresns.2012.02.005

Hoexter, M. Q., de Souza Duran, F. L., D’Alcante, C. C., Dougherty, D. D., Shavitt, R. G., Lopes, A. C., et al. (2012). Gray matter volumes in obsessivecompulsive disorder before and after fluoxetine or cognitive-behavior therapy: a randomized clinical trial. Contemp. Clin. Trials 37, 734-745. doi: 10. 1038/npp.2011.250

Hone-Blanchet, A., Edden, R. A., and Fecteau, S. (2015). Online effects of transcranial direct current stimulation in real time on human prefrontal and striatal metabolites. Biol. Psychiatry 80, 432-438. doi: 10.1016/j.biopsych.2015. 11.008

Hou, J., Song, L., Zhang, W., Wu, W., Wang, J., Zhou, D., et al. (2013). Morphologic and functional connectivity alterations of corticostriatal and default mode network in treatment-naive patients with obsessivecompulsive disorder. PLoS One 8:e83931. doi: 10.1371/journal.pone.00 83931
Hu, Y., Salmeron, B. J., Gu, H., Stein, E. A., and Yang, Y. (2015). Impaired functional connectivity within and between frontostriatal circuits and its association with compulsive drug use and trait impulsivity in cocaine addiction. JAMA Psychiatry 72, 584-592. doi: 10.1001/jamapsychiatry.2015.1

Hudson, J. I., Hiripi, E., Pope, H. G., and Kessler, R. C. (2007). The prevalence and correlates of eating disorders in the National comorbidity survey replication. Biol. Psychiatry 61, 348-358. doi: 10.1016/j.biopsych.2006. 03.040

Insel, T. R. (2014). The NIMH research domain criteria (RDoC) project: precision medicine for psychiatry. Am. J. Psychiatry 171, 395-397. doi: 10.1176/appi.ajp. 2014.14020138

Isserles, M., Shalev, A. Y., Roth, Y., Peri, T., Kutz, I., Zlotnick, E., et al. (2013). Effectiveness of deep transcranial magnetic stimulation combined with a brief exposure procedure in post-traumatic stress disorder-a pilot study. Brain Stimul. 6, 377-383. doi: 10.1016/j.brs.2012.07.008

Iwabuchi, S. J., Liddle, P. F., and Palaniyappan, L. (2015). Structural connectivity of the salience-executive loop in schizophrenia. Eur. Arch. Psychiatry Clin. Neurosci. 265, 163-166. doi: 10.1007/s00406-014-0547-z

Janes, A. C., Farmer, S., Peechatka, A. L., de B Frederick, B., and Lukas, S. E. (2015), Insula-dorsal anterior cingulate cortex coupling is associated with enhanced brain reactivity to smoking cues. Neuropsychopharmacology 40, 1561-1568. doi: 10.1038/npp.2015.9

Jiang, J., Beck, J., Heller, K., and Egner, T. (2015). An insula-frontostriatal network mediates flexible cognitive control by adaptively predicting changing control demands. Nat. Commun. 6:8165. doi: 10.1038/ncomms9165

Kapur, S. (2003). Psychosis as a state of aberrant salience: a framework linking biology, phenomenology and pharmacology in schizophrenia. Am. J. Psychiatry 160, 13-23. doi: 10.1176/appi.ajp.160.1.13

Karabanov, A., Ziemann, U., Hamada, M., George, M. S., Quartarone, A., Classen, J., et al. (2015). Consensus paper: probing homeostatic plasticity of human cortex with non-invasive transcranial brain stimulation. Brain Stimul. 8, 993-1006. doi: 10.1016/j.brs.2015.06.016

Ke, J., Zhang, L., Qi, R., Xu, Q., Li, W., Hou, C., et al. (2015). Altered blood oxygen level-dependent signal variability in chronic post-traumatic stress disorder during symptom provocation. Neuropsychiatr. Dis. Treat. 11, 1805-1815. doi: $10.2147 /$ NDT.s87332

Kennedy, S. H., Giacobbe, P., Rizvi, S. J., Placenza, F. M., Nishikawa, Y., Mayberg, H. S., et al. (2011). Deep brain stimulation for treatment-resistant depression: follow-up after 3 to 6 years. Am. J. Psychiatry 168, 502-510. doi: 10. 1176/appi.ajp.2010.10081187

Kerestes, R., Harrison, B. J., Dandash, O., Stephanou, K., Whittle, S., Pujol, J., et al. (2015). Specific functional connectivity alterations of the dorsal striatum in young people with depression. Neuroimage Clin. 7, 266-272. doi: 10.1016/j. nicl.2014.12.017

Knable, M. B., and Weinberger, D. R. (1997). Dopamine, the prefrontal cortex and schizophrenia. J. Psychopharmacol. 11, 123-131. doi: 10. 1177/026988119701100205

Knoch, D., Nitsche, M. A., Fischbacher, U., Eisenegger, C., Pascual-Leone, A., and Fehr, E. (2008). Studying the neurobiology of social interaction with transcranial direct current stimulation-The example of punishing unfairness. Cereb. Cortex 18, 1987-1990. doi: 10.1093/cercor/bhm237

Ko, J. H., Monchi, O., Ptito, A., Bloomfield, P., Houle, S., and Strafella, A. P. (2008). Theta burst stimulation-induced inhibition of dorsolateral prefrontal cortex reveals hemispheric asymmetry in striatal dopamine release during a set-shifting task: a TMS- $\left[{ }^{11} \mathrm{C}\right]$ raclopride PET study. Eur. J. Neurosci. 28, 2147-2155. doi: 10.1111/j.1460-9568.2008.06501.x

Koch, S. B. J., van Zuiden, M., Nawijn, L., Frijling, J. L., Veltman, D. J., and Olff, M. (2016). Aberrant resting-state brain activity in posttraumatic stress disorder: a meta-analysis and systematic review. Depress. Anxiety 33, 592-605. doi: 10. 1002/da.22478

Koehler, S., Ovadia-Caro, S., van der Meer, E., Villringer, A., Heinz, A., Romanczuk-Seiferth, N., et al. (2013). Increased functional connectivity between prefrontal cortex and reward system in pathological gambling. PLoS One 8:e84565. doi: 10.1371/journal.pone.0084565

Koob, G. F., and Volkow, N. D. (2010). Neurocircuitry of addiction. Neuropsychopharmacology 35, 217-238. doi: 10.1038/npp. 2009.110

Kreuzer, P. M., Schecklmann, M., Lehner, A., Wetter, T. C., Poeppl, T. B., Rupprecht, R., et al. (2015). The ACDC pilot trial: targeting the anterior 
cingulate by double cone coil rTMS for the treatment of depression. Brain Stimul. 8, 240-246. doi: 10.1016/j.brs.2014.11.014

Kroemer, N. B., Guevara, A., Ciocanea Teodorescu, I., Wuttig, F., Kobiella, A., and Smolka, M. N. (2014). Balancing reward and work: anticipatory brain activation in NAcc and VTA predict effort differentially. Neuroimage 102, 510-519. doi: 10.1016/j.neuroimage.2014.07.060

Kühn, A. A., and Volkmann, J. (2016). Innovations in deep brain stimulation methodology. Mov. Disord. doi: 10.1002/mds.26703 [Epub ahead of print].

Kuo, M.-F., and Nitsche, M. A. (2012). Effects of transcranial electrical stimulation on cognition. Clin. EEG Neurosci. 43, 192-199. doi: 10.1177/15500594124 44975

Lamichhane, B., Adhikari, B. M., and Dhamala, M. (2016). Salience network activity in perceptual decisions. Brain Connect. 6, 558-571. doi: 10.1089/brain. 2015.0392

Lao-Kaim, N. P., Fonville, L., Giampietro, V. P., Williams, S. C., Simmons, A., and Tchanturia, K. (2015). Aberrant function of learning and cognitive control networks underlie inefficient cognitive flexibility in anorexia nervosa: a cross- sectional fMRI study. PLoS One 10:e0124027. doi: 10.1371/journal.pone. 0124027

Lapenta, O. M., Sierve, K. D., de Macedo, E. C., Fregni, F., and Boggio, P. S. (2014). Transcranial direct current stimulation modulates ERP-indexed inhibitory control and reduces food consumption? Appetite 83, 42-48. doi: 10.1016/j. appet.2014.08.005

Lee, S. K., Chun, J. W., Lee, J. S., Park, H. J., Jung, Y. C., Seok, J. H., et al. (2014). Abnormal neural processing during emotional salience attribution of affective asymmetry in patients with schizophrenia. PLoS One 9:e90792. doi: 10. 1371/journal.pone.0090792

Lefaucheur, J. P., André-Obadia, N., Antal, A., Ayache, S. S., Baeken, C., Benninger, D. H., et al. (2014). Evidence-based guidelines on the therapeutic use of repetitive transcranial magnetic stimulation (rTMS). Clin. Neurophysiol. 125, 2150-2206. doi: 10.1016/j.clinph.2014.05.021

Lefebvre, S., Demeulemeester, M., Leroy, A., Delmaire, C., Lopes, R., Pins, D., et al. (2016). Network dynamics during the different stages of hallucinations in schizophrenia. Hum. Brain Mapp. 7, 2571-2586. doi: 10.1002/hbm. 23197

Liberzon, I., Taylor, S. F., Fig, L. M., and Koeppe, R. A. (1996/1997). Alteration of corticothalamic perfusion ratios during a PTSD flashback. Depress. Anxiety 4, 146-150. doi: 10.1002/(sici)1520-6394(1996)4:3<146::aid-da 9>3.0.co;2-e

Lin, H.-C., Pan, H.-C., Lin, S.-H., Lo, Y.-C., Shen, E. T.-H., Liao, L.-D., et al. (2016). Central thalamic deep-brain stimulation alters striatal-thalamic connectivity in cognitive neural behavior. Front. Neural Circuits 9:87. doi: 10.3389/fncir.2015. 00087

Lipsman, N., Giacobbe, P., and Lozano, A. M. (2013a). Deep brain stimulation in obsessive-compulsive disorder: neurocircuitry and clinical experience. Handb. Clin. Neurol. 116, 245-250. doi: 10.1016/B978-0-444-53497-2. 00019-X

Lipsman, N., Woodside, D. B., Giacobbe, P., Hamani, C., Carter, J. C., Norwood, S. J., et al. (2013b). Subcallosal cingulate deep brain stimulation for treatment-refractory anorexia nervosa: a phase 1 pilot trial. Lancet 381, 1361-1370. doi: 10.1016/S0140-6736(12)62188-6

Liston, C., Chen, A. C., Zebley, B. D., Drysdale, A. T., Gordon, R., Leuchter, B., et al. (2014). Default mode network mechanisms of transcranial magnetic stimulation in depression. Biol. Psychiatry 76, 517-526. doi: 10. 1016/j.biopsych.2014.01.023

Lozano, A. M., and Lipsman, N. (2013). Probing and regulating dysfunctional circuits using deep brain stimulation. Neuron 77, 406-424. doi: 10.1016/j. neuron.2013.01.020

Manelis, A., Almeida, J. R., Stiffler, R., Lockovich, J. C., Aslam, H. A., and Phillips, M. L. (2016). Anticipation-related brain connectivity in bipolar and unipolar depression: a graph theory approach. Brain 9, 2554-2566. doi: 10. 1093/brain/aww157

Manoliu, A., Riedl, V., Doll, A., Bäuml, J. G., Mühlau, M., Schwerthöffer, D., et al. (2013). Insular dysfunction reflects altered between-network connectivity and severity of negative symptoms in schizophrenia during psychotic remission. Front. Hum. Neurosci. 7:216. doi: 10.3389/fnhum.2013.00216

Mantovani, A., Simpson, H. B., Fallon, B. A., Rossi, S., and Lisanby, S. H. (2010). Randomized sham-controlled trial of repetitive transcranial magnetic stimulation in treatment-resistant obsessive-compulsive disorder.
Int. J. Neuropsychopharmacol. 2, 217-227. doi: 10.1017/S14611457099 90435

Marsh, R., Horga, G., Wang, Z., Wang, P., Klahr, K. W., Berner, L. A., et al. (2011). An FMRI study of self-regulatory control and conflict resolution in adolescents with bulimia nervosa. Am. J. Psychiatry 11, 1210-1220. doi: 10.1176/appi.ajp. 2011.11010094

Marsh, R., Maia, T. V., and Peterson, B. S. (2009a). Functional disturbances within frontostriatal circuits across multiple childhood psychopathologies. Am. J. Psychiatry 166, 664-674. doi: 10.1176/appi.ajp.2009.08091354

Marsh, R., Steinglass, J. E., Gerber, A. J., Graziano O'Leary, K., Wang, Z., Murphy, D., et al. (2009b). Deficient activity in the neural systems that mediate self-regulatory control in bulimia nervosa. Arch. Gen. Psychiatry 1, 51-63. doi: 10.1001/archgenpsychiatry.2008.504

Mayberg, H. S., Lozano, A. M., Voon, V., McNeely, H. E., Seminowicz, D., Hamani, C., et al. (2005). Deep brain stimulation for treatment-resistant depression. Neuron 45, 651-660. doi: 10.1016/j.neuron.2005.02.014

McClelland, J., Bozhilova, N., Campbell, I., and Schmidt, U. (2013). A systematic review of the effects of neuromodulation on eating and body weight: evidence from human and animal studies. Eur. Eat. Disord. Rev. 21, 436-455. doi: 10. 1002/erv.2256

McGirr, A., Karmani, S., Arsappa, R., Berlim, M. T., Thirthalli, J., Muralidharan, K., et al. (2016). Clinical efficacy and safety of repetitive transcranial magnetic stimulation in acute bipolar depression. World Psychiatry 1, 85-86. doi: 10.1002/wps.20300

McTeague, L. M., Goodkind, M. S., and Etkin, A. (2016). Transdiagnostic impairment of cognitive control in mental illness. J. Psychiatr. Res. 83, 37-46. doi: 10.1016/j.jpsychires.2016.08.001

Meng, L., Jiang, J., Jin, C., Liu, J., Zhao, Y., Wang, W., et al. (2016). Traumaspecific grey matter alterations in PTSD. Sci. Rep. 6:33748. doi: 10.1038/srep 33748

Menon, V. (2011). Large-scale brain networks and psychopathology: a unifying triple network model. Trends Cogn. Sci. 15, 483-506. doi: 10.1016/j.tics.2011. 08.003

Menon, V., and Uddin, L. Q. (2010). Saliency, switching, attention and control: a network model of insula function. Brain Struct. Funct. 214, 655-667. doi: 10. 1007/s00429-010-0262-0

Meron, D., Hedger, N., Garner, M., and Baldwin, D. S. (2015). Transcranial direct current stimulation (tDCS) in the treatment of depression: systematic review and meta- analysis of efficacy and tolerability. Neurosci. Biobehav. Rev. 57, 46-62. doi: 10.1016/j.neubiorev.2015.07.012

Metzger, C. D., Eckert, U., Steiner, J., Sartorius, A., Buchmann, J. E., Stadler, J., et al. (2010). High field FMRI reveals thalamocortical integration of segregated cognitive and emotional processing in mediodorsal and intralaminar thalamic nuclei. Front. Neuroanat. 4:138. doi: 10.3389/fnana.2010. 00138

Mikolas, P., Melicher, T., Skoch, A., Matejka, M., Slovakova, A., Bakstein, E., et al. (2016). Connectivity of the anterior insula differentiates participants with first-episode schizophrenia spectrum disorders from controls: a machinelearning study. Psychol. Med. 46, 2695-2704. doi: 10.1017/s0033291716000878

Milev, R. V., Giacobbe, P., Kennedy, S. H., Blumberger, D. M., Daskalakis, Z. J. Downar, J., et al. (2016). Canadian network for mood and anxiety treatments (CANMAT) 2016 clinical guidelines for the management of adults with major depressive disorder: section 4. neurostimulation treatments. Can. J. Psychiatry 61, 561-575. doi: 10.1177/0706743716660033

Mir-Moghtadaei, A., Caballero, R., Fried, P., Fox, M. D., Lee, K., Giacobbe, P., et al. (2015). Concordance between BeamF3 and MRI-neuronavigated target sites for repetitive transcranial magnetic stimulation of the left dorsolateral prefrontal cortex. Brain Stimul. 5, 965-973. doi: 10.1016/j.brs.2015.05.008

Mitchell, A. S. (2015). The mediodorsal thalamus as a higher order thalamic relay nucleus important for learning and decision-making. Neurosci. Biobehav. Rev. 54, 76-88. doi: 10.1016/j.neubiorev.2015.03.001

Morales, A. M., Kohno, M., Robertson, C. L., Dean, A. C., Mandelkern, M. A., and London, E. D. (2015). Gray-matter volume, midbrain dopamine D2/D3 receptors and drug craving in methamphetamine users. Mol. Psychiatry 20, 764-771. doi: 10.1038/mp.2015.47

Moran, L. V., Tagamets, M. A., Sampath, H., O'Donnell, A., Stein, E. A., Kochunov, P., et al. (2013). Disruption of anterior insula modulation of largescale brain networks in schizophrenia. Biol. Psychiatry 74, 467-474. doi: 10. 1016/j.biopsych.2013.02.029 
Moreno-López, L., Catena, A., Fernández-Serrano, M. J., Delgado-Rico, E., Stamatakis, E. A., Pérez-García, M., et al. (2012). Trait impulsivity and prefrontal gray matter reductions in cocaine dependent individuals. Drug Alcohol Depend. 125, 208-214. doi: 10.1016/j.drugalcdep.2012.02.012

Morey, R. A., Dunsmoor, J. E., Haswell, C. C., Brown, V. M., Vora, A., Weiner, J., et al. (2015). Fear learning circuitry is biased toward generalization of fear associations in posttraumatic stress disorder. Transl. Psychiatry 5:e700. doi: 10. 1038/tp.2015.196

Morishita, T., Fayad, S. M., Higuchi, M. A., Nestor, K. A., and Foote, K. D. (2014). Deep brain stimulation for treatment-resistant depression: systematic review of clinical outcomes. Neurotherapeutics 11, 475-484. doi: 10.1007/s13311-0140282-1

Morris, S. E., and Cuthbert, B. N. (2012). Research domain criteria: cognitive systems, neural circuits, and dimensions of behavior. Dialogues Clin. Neurosci. $14,29-37$.

Mueller, S. G., Ng, P., Neylan, T., Mackin, S., Wolkowitz, O., Mellon, S., et al. (2015). Evidence for disrupted gray matter structural connectivity in posttraumatic stress disorder. Psychiatry Res. 234, 194-201. doi: 10.1016/j. pscychresns.2015.09.006

Nakamae, T., Sakai, Y., Abe, Y., Nishida, S., Fukui, K., Yamada, K., et al. (2014). Altered fronto-striatal fiber topography and connectivity in obsessivecompulsive disorder. PLoS One 9:e112075. doi: 10.1371/journal.pone.01 12075

Naqvi, N. H., and Bechara, A. (2009). The hidden island of addiction: the insula. Trends Neurosci. 32, 56-67. doi: 10.1016/j.tins.2008.09.009

Nekovarova, T., Fajnerova, I., Horacek, J., and Spaniel, F. (2014). Bridging disparate symptoms of schizophrenia: a triple network dysfunction theory. Front. Behav. Neurosci. 8:171. doi: 10.3389/fnbeh.2014. 00171

Nitsche, M. A., Cohen, L. G., Wassermann, E. M., Priori, A., Lang, N., Antal, A., et al. (2008). Transcranial direct current stimulation: state of the art 2008. Brain Stimul. 1, 206-223. doi: 10.1016/j.brs.2008.06.004

Noda, Y., Silverstein, W. K., Barr, M. S., Vila-Rodriguez, F., Downar, J., Rajji, T. K., et al. (2015). Neurobiological mechanisms of repetitive transcranial magnetic stimulation of the dorsolateral prefrontal cortex in depression: a systematic review. Psychol. Med. 45, 3411-3432. doi: 10.1017/S0033291715001609

Palaniyappan, L., Mallikarjun, P., Joseph, V., White, T. P., and Liddle, P. F. (2011). Reality distortion is related to the structure of the salience network in schizophrenia. Psychol. Med. 41, 1701-1708. doi: 10.1017/S0033291710002205

Palaniyappan, L., Simmonite, M., White, T. P., Liddle, E. B., and Liddle, P. F. (2013). Neural primacy of the salience processing system in schizophrenia. Neuron 79, 814-828. doi: 10.1016/j.neuron.2013.06.027

Park, S. Q., Kahnt, T., Beck, A., Cohen, M. X., Dolan, R. J., Wrase, J., et al. (2010). Prefrontal cortex fails to learn from reward prediction errors in alcohol dependence. J. Neurosci. 30, 7749-7753. doi: 10.1523/JNEUROSCI.5587-09. 2010

Parnaudeau, S., O’Neill, P. K., Bolkan, S. S., Ward, R. D., Abbas, A. I., Roth, B. L., et al. (2013). Inhibition of mediodorsal thalamus disrupts thalamofrontal connectivity and cognition. Neuron 77, 1151-1162. doi: 10.1016/j.neuron.2013. 01.038

Pascual-Leone, A., Rubio, B., Pallardó, F., and Catalá, M. D. (1996). Rapidrate transcranial magnetic stimulation of left dorsolateral prefrontal cortex in drug-resistant depression. Lancet 348, 233-237. doi: 10.1016/s01406736(96)01219-6

Patel, R., Spreng, R. N., Shin, L. M., and Girard, T. A. (2012). Neurocircuitry models of posttraumatic stress disorder and beyond: a meta-analysis of functional neuroimaging studies. Neurosci. Biobehav. Rev. 36, 2130-2142. doi: 10.1016/j.neubiorev.2012.06.003

Paulus, M. P., and Stein, M. B. (2006). An insular view of anxiety. Biol. Psychiatry 60, 383-387. doi: 10.1016/j.biopsych.2006.03.042

Peper, J. S., Mandl, R. C. W., Braams, B. R., de Water, E., Heijboer, A. C., Koolschijn, P. C. M. P., et al. (2013). Delay discounting and frontostriatal fiber tracts: a combined DTI and MTR study on impulsive choices in healthy young adults. Cereb. Cortex 23, 1695-1702. doi: 10.1093/cercor/bhs163

Perera, T., George, M. S., Grammer, G., Janicak, P. G., Pascual-Leone, A., and Wirecki, T. S. (2016). The clinical TMS society consensus review and treatment recommendations for TMS therapy for major depressive disorder. Brain Stimul. 3, 336-346. doi: 10.1016/j.brs.2016.03.010
Peters, J., and Büchel, C. (2011). The neural mechanisms of inter-temporal decision-making: understanding variability. Trends Cogn. Sci. 15, 227-239. doi: 10.1016/j.tics.2011.03.002

Peterson, A., Thome, J., Frewen, P., and Lanius, R. A. (2014). Resting-state neuroimaging studies: a new way of identifying differences and similarities among the anxiety disorders? Can. J. Psychiatry 59, 294-300.

Polanía, R., Paulus, W., and Nitsche, M. A. (2012). Modulating corticostriatal and thalamo-cortical functional connectivity with transcranial direct current stimulation. Hum. Brain Mapp. 33, 2499-2508. doi: 10.1002/hbm. 21380

Pu, W., Li, L., Zhang, H., Ouyang, X., Liu, H., Zhao, J., et al. (2012). Morphological and functional abnormalities of salience network in the early-stage of paranoid schizophrenia. Schizophr. Res. 141, 15-21. doi: 10.1016/j.schres.2012. 07.017

Pujol, N., Penadés, R., Rametti, G., Catalán, R., Vidal-Piñeiro, D., Palacios, E., et al. (2013). Inferior frontal and insular cortical thinning is related to dysfunctional brain activation/deactivation during working memory task in schizophrenic patients. Psychiatry Res. 214, 94-9101. doi: 10.1016/j.pscychresns.2013. 06.008

Qiu, Y., Lv, X., Su, H., Jiang, G., Tian, J., Zhuo, F., et al. (2013). Reduced regional homogeneity in bilateral frontostriatal system relates to higher impulsivity behavior in codeine-containing cough syrups dependent individuals. PLoS One 8:e78738. doi: 10.1371/journal.pone.0078738

Quan, M., Lee, S. H., Kubicki, M., Kikinis, Z., Rathi, Y., Seidman, L. J., et al. (2013). White matter tract abnormalities between rostral middle frontal gyrus, inferior frontal gyrus and striatum in first-episode schizophrenia. Schizophr. Res. 145, 1-10. doi: 10.1016/j.schres.2012.11.028

Rachid, F. (2016). Neurostimulation techniques in the treatment of nicotine dependence: a review. Am. J. Addict. 25, 436-451. doi: 10.1111/ajad. 12405

Raichle, M. E. (2015). The brain's default mode network. Annu. Rev. Neurosci. 38, 433-447. doi: 10.1146/annurev-neuro-071013-014030

Rauch, S. L., Dougherty, D. D., Malone, D., Rezai, A., Friehs, G., Fischman, A. J., et al. (2006a). A functional neuroimaging investigation of deep brain stimulation in patients with obsessive-compulsive disorder. J. Neurosurg. 104, 558-565. doi: 10.3171/jns.2006.104.4.558

Rauch, S. L., Shin, L. M., and Phelps, E. A. (2006b). Neurocircuitry models of posttraumatic stress disorder and extinction: human neuroimaging researchpast, present and future. Biol. Psychiatry 60, 376-382. doi: 10.1016/j.biopsych. 2006.06.004

Rothemund, Y., Buchwald, C., Georgiewa, P., Bohner, G., Bauknecht, H.-C., Ballmaier, M., et al. (2011). Compulsivity predicts fronto striatal activation in severely anorectic individuals. Neuroscience 197, 242-250. doi: 10.1016/j. neuroscience.2011.09.016

Rush, A. J., Trivedi, M. H., Wisniewski, S. R., Nierenberg, A. A., Stewart, J. W., Warden, D., et al. (2006). Acute and longer-term outcomes in depressed outpatients requiring one or several treatment steps: a STAR*D report. Am. J. Psychiatry 163, 1905-1917. doi: 10.1176/ajp.2006.163.11.1905

Sale, M. V., Mattingley, J. B., Zalesky, A., and Cocchi, L. (2015). Imaging human brain networks to improve the clinical efficacy of non-invasive brain stimulation. Neurosci. Biobehav. Rev. 57, 187-198. doi: 10.1016/j.neubiorev. 2015.09.010

Salomons, T. V., Dunlop, K., Kennedy, S. H., Flint, A., Geraci, J., Giacobbe, P., et al. (2014). Resting-state cortico-thalamic-striatal connectivity predicts response to dorsomedial prefrontal rTMS in major depressive disorder. Neuropsychopharmacology 39, 488-498. doi: 10.1038/npp.2013.222

Sanders, N., Smeets, P. A., van Elburg, A. A., Danner, U. N., van Meer, F., Hoek, H. W., et al. (2015). Altered food-cue processing in chronically ill and recovered women with anorexia nervosa. Front. Behav. Neurosci. 9:46. doi: 10. 3389/fnbeh.2015.00046

Schäfer, A., Vaitl, D., and Schienle, A. (2010). Regional grey matter volume abnormalities in bulimia nervosa and binge-eating disorder. Neuroimage 50, 639-643. doi: 10.1016/j.neuroimage.2009.12.063

Schienle, A., Schäfer, A., Hermann, A., and Vaitl, D. (2009). Binge-eating disorder: reward sensitivity and brain activation to images of food. Biol. Psychiatry 65, 654-661. doi: 10.1016/j.biopsych.2008.09.028

Schiller, C. E., Minkel, J., Smoski, M. J., and Dichter, G. S. (2013). Remitted major depression is characterized by reduced prefrontal cortex reactivity 
to reward loss. J. Affect. Disord. 151, 756-762. doi: 10.1016/j.jad.2013. 06.016

Schlaepfer, T. E., Bewernick, B. H., Kayser, S., Hurlemann, R., and Coenen, V. A. (2014). Deep brain stimulation of the human reward system for major depression-rationale, outcomes and outlook. Neuropsychopharmacology 39, 1303-1314. doi: 10.1038/npp.2014.28

Schultz, W., Tremblay, L., and Hollerman, J. R. (2003). Changes in behaviorrelated neuronal activity in the striatum during learning. Trends Neurosci. 26, 321-328. doi: 10.1016/S0166-2236(03)00122-x

Seeley, W. W., Menon, V., Schatzberg, A. F., Keller, J., Glover, G. H., Kenna, H., et al. (2007). Dissociable intrinsic connectivity networks for salience processing and executive control. J. Neurosci. 27, 2349-2356. doi: 10.1523/JNEUROSCI. 5587-06.2007

Shepherd, G. M. G. (2013). Corticostriatal connectivity and its role in disease. Nat. Rev. Neurosci. 14, 278-291. doi: 10.1038/nrn3469

Shott, M. E., Pryor, T. L., Yang, T. T., and Frank, G. K. (2016). Greater insula white matter fiber connectivity in women recovered from anorexia nervosa. Neuropsychopharmacology 41, 498-507. doi: 10.1038/npp. 2015.172

Skunde, M., Walther, S., Simon, J. J., Wu, M., Bendszus, M., Herzog, W., et al. (2016). Neural signature of behavioural inhibition in women with bulimia nervosa. J. Psychiatry Neurosci. 41, E69-E78. doi: 10.1503/jpn. 150335

Smoski, M. J., Rittenberg, A., and Dichter, G. S. (2011). Major depressive disorder is characterized by greater reward network activation to monetary than pleasant image rewards. Psychiatry Res. 194, 263-270. doi: 10.1016/j.pscychresns.2011. 06.012

Sommer, I. E. C., Diederen, K. M. J., Blom, J. D., Willems, A., Kushan, L., Slotema, K., et al. (2008). Auditory verbal hallucinations predominantly activate the right inferior frontal area. Brain 131, 3169-3177. doi: 10. 1093/brain/awn251

Stefurak, T., Mikulis, D., Mayberg, H., Lang, A. E., Hevenor, S., Pahapill, P., et al. (2003). Deep brain stimulation for Parkinson's disease dissociates mood and motor circuits: a functional MRI case study. Mov. Disord. 18, 1508-1516. doi: $10.1002 / \mathrm{mds} .10593$

Stoy, M., Schlagenhauf, F., Sterzer, P., Bermpohl, F., Hägele, C., Suchotzki, K., et al. (2011). Hyporeactivity of ventral striatum towards incentive stimuli in unmedicated depressed patients normalizes after treatment with escitalopram. J. Psychopharmacol. 26, 677-688. doi: 10.1177/0269881111416686

Strafella, A. P., Paus, T., Barrett, J., and Dagher, A. (2001). Repetitive transcranial magnetic stimulation of the human prefrontal cortex induces dopamine release in the caudate nucleus. J. Neurosci. 21:RC157.

Strafella, A. P., Paus, T., Fraraccio, M., and Dagher, A. (2003). Striatal dopamine release induced by repetitive transcranial magnetic stimulation of the human motor cortex. Brain 126, 2609-2615. doi: 10.1093/brain/awg268

Sun, J., Chen, Q., Zhang, Q., Li, Y., Li, H., Wei, D., et al. (2016). Training your brain to be more creative: brain functional and structural changes induced by divergent thinking training. Hum. Brain Mapp. 37, 3375-3387. doi: 10 . $1002 / \mathrm{hbm} .23246$

Tomasi, D., and Volkow, N. D. (2013). Striatocortical pathway dysfunction in addiction and obesity: differences and similarities. Crit. Rev. Biochem. Mol. Biol. 48, 1-19. doi: 10.3109/10409238.2012.735642

Tortella, G., Casati, R., Aparicio, L. V., Mantovani, A., Senço, N., D'Urso, G., et al. (2015). Transcranial direct current stimulation in psychiatric disorders. World J. Psychiatry 5, 88-8102. doi: 10.5498/wjp.v5.i1.88

Trevizol, A. P., Shiozawa, P., Cook, I. A., Sato, I. A., Kaku, C. B., Guimarães, F. B., et al. (2016). Transcranial magnetic stimulation for obsessive-compulsive disorder: an updated systematic review and meta-analysis. J. ECT 32, 262-266. doi: $10.1097 /$ yct.0000000000000335

Uddin, L. Q. (2017). Salience Network of the Human Brain. San Diego: Academic Press. Available online at: http://dx.doi.org/10.1016/B978-0-12804593-0.00007-2, 34 .

Vaghi, M. M., Vértes, P. E., Kitzbichler, M. G., Apergis-Schoute, A. M., van der Flier, F. E., Fineberg, N. A., et al. (2016). Specific frontostriatal circuits for impaired cognitive flexibility and goal-directed planning in obsessivecompulsive disorder: evidence from resting-state functional connectivity. Biol. Psychiatry doi: 10.1016/j.biopsych.2016.08.009 [Epub ahead of print].

Val-Laillet, D., Aarts, E., Weber, B., Ferrari, M., Quaresima, V., Stoeckel, L. E., et al. (2015). Neuroimaging and neuromodulation approaches to study eating behavior and prevent and treat eating disorders and obesity. Neuroimage Clin. 8, 1-31. doi: 10.1016/j.nicl.2015.03.016

Van den Eynde, F., Suda, M., Broadbent, H., Guillaume, S., Van den Eynde, M., Steiger, H., et al. (2012). Structural magnetic resonance imaging in eating disorders: a systematic review of voxel-based morphometry studies. Eur. Eat. Disord. Rev. 20, 94-105. doi: 10.1002/erv.1163

Veit, R., Singh, V., Sitaram, R., Caria, A., Rauss, K., and Birbaumer, N. (2012). Using real-time fmri to learn voluntary regulation of the anterior insula in the presence of threat-related stimuli. Soc. Cogn. Affect. Neurosci. 7, 623-634. doi: $10.1093 /$ scan/nsr061

Vollstädt-Klein, S., Wichert, S., Rabinstein, J., Buhler, M., Klein, O., Ende, O., et al. (2010). Initial, habitual and compulsive alcohol use is characterized by a shift of cue processing from ventral to dorsal striatum. Addiction 105, 1741-1749. doi: $10.1111 / j .1360-0443.2010 .03022 . x$

Voss, U., Holzmann, R., Hobson, A., Paulus, W., Koppehele-Gossel, J., Klimke, A., et al. (2014). Induction of self awareness in dreams through frontal low current stimulation of gamma activity. Nat. Neurosci. 17, 810-812. doi: 10.1038/nn. 3719

Walsh, E., Carl, H., Eisenlohr-Moul, T., Minkel, J., Crowther, A., Moore, T., et al. (2016). Attenuation of frontostriatal connectivity during reward processing predicts response to psychotherapy in major depressive disorder. Neuropsychopharmacology doi: 10.1038/npp.2016.179 [Epub ahead of print].

Wang, C., Ji, F., Hong, Z., Poh, J. S., Krishnan, R., Lee, J., et al. (2016). Disrupted salience network functional connectivity and white-matter microstructure in persons at risk for psychosis: findings from the LYRIKS study. Psychol. Med. 46, 2771-2783. doi: 10.1017/s0033291716001410

Wang, X., Zhang, W., Sun, Y., Hu, M., and Chen, A. (2016). Aberrant intrasalience network dynamic functional connectivity impairs large-scale network interactions in schizophrenia. Neuropsychologia 93, 262-270. doi: 10.1016/j. neuropsychologia.2016.11.003

Webb, C. A., Dillon, D. G., Pechtel, P., Goer, F. K., Murray, L., Huys, Q. J., et al. (2016). Neural correlates of three promising endophenotypes of depression: evidence from the EMBARC study. Neuropsychopharmacology 41, 454-463. doi: 10.1038/npp.2015.165

Webb, C. A., Weber, M., Mundy, E. A., and Killgore, W. D. (2014). Reduced gray matter volume in the anterior cingulate, orbitofrontal cortex and thalamus as a function of mild depressive symptoms: a voxel-based morphometric analysis. Psychol. Med. 44, 2833-2843. doi: 10.1017/s00332917140 00348

Weber, M. J., Messing, S. B., Rao, H., Detre, J. A., and ThompsonSchill, S. L. (2014). Prefrontal transcranial direct current stimulation alters activation and connectivity in cortical and subcortical reward systems: a tDCS-fMRI study. Hum. Brain Mapp. 35, 3673-3686. doi: 10.1002/hbm. 22429

White, S. F., Costanzo, M. E., Blair, J. R., and Roy, M. J. (2014). PTSD symptom severity is associated with increased recruitment of top-down attentional control in a trauma-exposed sample. Neuroimage Clin. 7, 19-27. doi: 10.1016/j. nicl.2014.11.012

White, T. P., Joseph, V., Francis, S. T., and Liddle, P. F. (2010). Aberrant salience network (bilateral insula and anterior cingulate cortex) connectivity during information processing in schizophrenia. Schizophr. Res. 123, 105-115. doi: 10. 1016/j.schres.2010.07.020

Whiteford, H. A., Degenhardt, L., Rehm, J., Baxter, A. J., Ferrari, A. J., Erskine, H. E., et al. (2013). Global burden of disease attributable to mental and substance use disorders: findings from the Global Burden of Disease Study 2010. Lancet 382, 1575-1586. doi: 10.1016/S0140-6736(13) 61611-6

Whiteford, H. A., Ferrari, A. J., Degenhardt, L., Feigin, V., and Vos, T. (2015). The global burden of mental, neurological and substance use disorders: an analysis from the Global Burden of Disease Study 2010. PLoS One 2:e0116820. doi: 10. 1371/journal.pone.0116820

Wierenga, C. E., Ely, A., Bischoff-Grethe, A., Bailer, U. F., Simmons, A. N., and Kaye, W. H. (2014). Are extremes of consumption in eating disorders related to an altered balance between reward and inhibition? Front. Behav. Neurosci. 8:410. doi: 10.3389/fnbeh.2014.00410

Williams, N. R., and Okun, M. S. (2013). Deep brain stimulation (DBS) at the interface of neurology and psychiatry. J. Clin. Invest. 123, 4546-4556. doi: 10. 1172/JCI68341 
Yarkoni, T., Poldrack, R. A., Nichols, T. E., Van Essen, D. C., and Wager, T. D. (2011). Large-scale automated synthesis of human functional neuroimaging data. Nat. Methods 8, 665-670. doi: 10.1038/nmeth.1635

Yeh, Y. W., Ho, P. S., Kuo, S. C., Chen, C. Y., Liang, C. S., Yen, C. H., et al. (2015). Disproportionate reduction of serotonin transporter may predict the response and adherence to antidepressants in patients with major depressive disorder: a positron emission tomography study with 4-[ ${ }^{18}$ F]-ADAM. Int. J. Neuropsychopharmacol. 7:pyu120. doi: 10.1093/ijnp/ pyu 120

Yeo, B. T. T., Krienen, F. M., Sepulcre, J., Sabuncu, M. R., Lashkari, D., Hollinshead, M., et al. (2011). The organization of the human cerebral cortex estimated by intrinsic functional connectivity. J. Neurophysiol. 106, 1125-1165. doi: 10.1152/jn.00338.2011

Yin, Y., Jin, C., Hu, X., Duan, L., Li, Z., Song, M., et al. (2011). Altered restingstate functional connectivity of thalamus in earthquake-induced posttraumatic stress disorder: a functional magnetic resonance imaging study. Brain Res. 1411, 98-107. doi: 10.1016/j.brainres.2011.07.016

Yuan, K., Yu, D., Bi, Y., Li, Y., Guan, Y., Liu, J., et al. (2016). The implication of frontostriatal circuits in young smokers: a resting-state study. Hum. Brain Mapp. 37, 2013-2026. doi: 10.1002/hbm.23153
Zhao, Y. J., Du, M. Y., Huang, X. Q., Lui, S., Chen, Z. Q., Liu, J., et al. (2014). Brain grey matter abnormalities in medication-free patients with major depressive disorder: a meta- analysis. Psychol. Med. 44, 2927-2937. doi: 10. 1017/S0033291714000518

Zuo, C., Ma, Y., Sun, B., Peng, S., Zhang, H., Eidelberg, D., et al. (2013). Metabolic imaging of bilateral anterior capsulotomy in refractory obsessive compulsive disorder: an FDG PET study. J. Cereb. Blood Flow Metab. 33, 880-887. doi: 10. 1038/jcbfm.2013.23

Conflict of Interest Statement: The authors declare that the research was conducted in the absence of any commercial or financial relationships that could be construed as a potential conflict of interest.

Copyright (c) 2016 Peters, Dunlop and Downar. This is an open-access article distributed under the terms of the Creative Commons Attribution License (CC BY). The use, distribution and reproduction in other forums is permitted, provided the original author(s) or licensor are credited and that the original publication in this journal is cited, in accordance with accepted academic practice. No use, distribution or reproduction is permitted which does not comply with these terms. 